\title{
Top terms of polynomial traces in Kra's plumbing construction
}

\author{
SARA MALONI \\ CAROLINE SERIES
}

Let $\Sigma$ be a surface of negative Euler characteristic together with a pants decomposition $\mathcal{P}$. Kra's plumbing construction endows $\Sigma$ with a projective structure as follows. Replace each pair of pants by a triply punctured sphere and glue, or "plumb", adjacent pants by gluing punctured disk neighbourhoods of the punctures. The gluing across the $i$-th pants curve is defined by a complex parameter $\tau_{i} \in \mathbb{C}$. The associated holonomy representation $\rho: \pi_{1}(\Sigma) \rightarrow \operatorname{PSL}(2, \mathbb{C})$ gives a projective structure on $\Sigma$ which depends holomorphically on the $\tau_{i}$. In particular, the traces of all elements $\rho(\gamma), \gamma \in \pi_{1}(\Sigma)$, are polynomials in the $\tau_{i}$.

Generalising results proved by Keen and the second author [4; 13] for the once and twice punctured torus respectively, we prove a formula giving a simple linear relationship between the coefficients of the top terms of $\rho(\gamma)$, as polynomials in the $\tau_{i}$, and the Dehn-Thurston coordinates of $\gamma$ relative to $\mathcal{P}$.

This will be applied in a later paper [7] by the first author to give a formula for the asymptotic directions of pleating rays in the Maskit embedding of $\Sigma$ as the bending measure tends to zero.

57M50; 30F40

\section{Introduction}

Let $\Sigma$ be a surface of negative Euler characteristic together with a pants decomposition $\mathcal{P}$. Kra's plumbing construction endows $\Sigma$ with a projective structure as follows. Replace each pair of pants by a triply punctured sphere and glue, or "plumb", adjacent pants by gluing punctured disk neighbourhoods of the punctures. The gluing across the $i$-th pants curve is defined by a complex parameter $\tau_{i} \in \mathbb{C}$. More precisely, $z w=\tau_{i}$ where $z, w$ are standard holomorphic coordinates on punctured disk neighbourhoods of the two punctures. The associated holonomy representation $\rho: \pi_{1}(\Sigma) \rightarrow \operatorname{PSL}(2, \mathbb{C})$ gives a projective structure on $\Sigma$ which depends holomorphically on the $\tau_{i}$, and in which the pants curves themselves are automatically parabolic. In particular, the traces of all elements $\rho(\gamma), \gamma \in \pi_{1}(\Sigma)$, are polynomials in the $\tau_{i}$. 
The main result of this paper is a very simple relationship between the coefficients of the top terms of $\rho(\gamma)$, as polynomials in the $\tau_{i}$, and the Dehn-Thurston coordinates of $\gamma$ relative to $\mathcal{P}$. This generalises results of Keen and the second author [4; 13] for the once and the twice punctured torus respectively.

Our formula is as follows. Let $\mathcal{S}$ denote the set of homotopy classes of multiple loops on $\Sigma$, and let the pants curves defining $\mathcal{P}$ be $\sigma_{i}, i=1, \ldots, \xi$. For brevity we usually refer to elements of $\mathcal{S}$ as curves (even if they are not connected); see Section 2. The Dehn-Thurston coordinates of $\gamma \in \mathcal{S}$ are $\mathbf{i}(\gamma)=\left(q_{i}, p_{i}\right), i=1, \ldots, \xi$, where $q_{i}=i\left(\gamma, \sigma_{i}\right) \in \mathbb{N} \cup\{0\}$ is the geometric intersection number between $\gamma$ and $\sigma_{i}$ and $p_{i} \in \mathbb{Z}$ is the twist of $\gamma$ about $\sigma_{i}$. We prove:

Theorem A Let $\gamma$ be a connected simple closed curve on $\Sigma$, not parallel to any of the pants curves $\sigma_{i}$. Then $\operatorname{Tr} \rho(\gamma)$ is a polynomial in $\tau_{1}, \ldots, \tau_{\xi}$ whose top terms are given by

where

$$
\begin{aligned}
\operatorname{Tr} \rho(\gamma) & = \pm i^{q} 2^{h}\left(\tau_{1}+\frac{\left(p_{1}-q_{1}\right)}{q_{1}}\right)^{q_{1}} \cdots\left(\tau_{\xi}+\frac{\left(p_{\xi}-q_{\xi}\right)}{q_{\xi}}\right)^{q_{\xi}}+R \\
& = \pm i^{q} 2^{h}\left(\tau_{1}^{q_{1}} \cdots \tau_{\xi}^{q_{\xi}}+\sum_{i=1}^{\xi}\left(p_{i}-q_{i}\right) \tau_{1}^{q_{1}} \cdots \tau_{i}^{q_{i}-1} \cdots \tau_{\xi}^{q_{\xi}}\right)+R
\end{aligned}
$$

- $q=\sum_{i=1}^{\xi} q_{i}>0$

- $R$ represents terms with total degree in $\tau_{1} \cdots \tau_{\xi}$ at most $q-2$ and of degree at most $q_{i}$ in the variable $\tau_{i}$;

- $h=h(\gamma)$ is the total number of scc-arcs in the standard representation of $\gamma$ relative to $\mathcal{P}$; see below.

If $q=0$, then $\gamma=\sigma_{i}$ for some $i, \rho(\gamma)$ is parabolic, and $\operatorname{Tr} \rho(\gamma)= \pm 2$.

The non-negative integer $h=h(\gamma)$ is defined as follows. The curve $\gamma$ is first arranged to intersect each pants curve minimally. In this position, it intersects a pair of pants $P$ in a number of arcs joining boundary loops of $P$. We call one of these an scc-arc (short for same-(boundary)-component-connector, called an archetype by Penner [12]) if it joins one boundary component to itself, and denote by $h$ the total number of scc-arcs, taken over all pants in $\mathcal{P}$.

The precise definition of the twist coordinates $p_{i}$ in Theorem A requires some care; we use essentially the standard definition implied in Fathi, Laudenbach and Poenaru [3] and explained in detail by D P Thurston [14] (called here the DT-twist; see Section 3.1), 
although for the proof we find useful the form given by Penner [12] (called here the P-twist and denoted $\hat{p}_{i}$; see Section 3.2).

We remark that the formula in Theorem A could of course be made neater by replacing the parameter $\tau$ by $\tau-1$; we use $\tau$ to be in accordance with the conventions of [4; 13]; see also Section 4.2.

We believe the formula in Theorem A noteworthy in its own right. However the main motivation for this work was the following. If the representation $\rho$ constructed in the above manner is free and discrete, then the resulting hyperbolic 3-manifold $M=\mathbb{H}^{3} / \rho\left(\pi_{1}(\Sigma)\right)$ lies on the boundary of quasifuchsian space $\mathcal{Q F}(\Sigma)$. One end of $M$ consists of a union of triply punctured spheres obtained by pinching in $\Sigma$ the curves $\sigma_{i}$ defining $\mathcal{P}$. Suppose that, in addition, $\rho\left(\pi_{1}(\Sigma)\right)$ is geometrically finite and that the other end $\Omega^{+} / \rho\left(\pi_{1}(\Sigma)\right)$ of $M$ is a Riemann surface homeomorphic to $\Sigma$. Since the triply punctured spheres are rigid, it follows from Ahlfors-Bers' measurable Riemann mapping theorem that the Riemann surface structure of $\Omega^{+} / \rho\left(\pi_{1}(\Sigma)\right)$ runs over the Teichmüller space $\mathcal{T}(\Sigma)$ of $\Sigma$. The image of the space of all such groups in the character variety $\mathcal{R}$ of $\Sigma$ is called the Maskit embedding of $\mathcal{T}(\Sigma)$.

In $[4 ; 13]$, special cases of the trace formula were important in constructing a computational method of locating the image $\mathcal{M}$ of $\mathcal{T}(\Sigma)$ in $\mathcal{R}$. In those papers we defined a pleating ray to be a line in $\mathcal{R}$ along which the projective class of the bending measure was kept constant. The trace formulae enabled us to find the asymptotic directions of pleating rays in $\mathcal{M}$ as the bending measure tends to zero. Theorem A allows the extension of these results to the general case; see Maloni [7].

The plan of this paper is as follows. After establishing preliminaries in Section 2, in Section 3 we review the Dehn-Thurston coordinates and, in particular, the definition of twists. In Section 4 we discuss the gluing construction which leads to the family of projective structures and their holonomy representation. In Section 5 we explain in detail the holonomy representation in various special cases, starting with arcs in a single pair of pants and going on to the one holed torus and four holed sphere. Finally, in Section 6, we make explicit the general combinatorial pattern of matrix products obtained in the holonomy, and use this to give an inductive proof of Theorem A.

\section{Background and notation}

Suppose $\Sigma$ is a surface of finite type, let $\mathcal{S}_{0}=\mathcal{S}_{0}(\Sigma)$ denote the set of free homotopy classes of connected closed simple non-boundary parallel curves on $\Sigma$, and let $\mathcal{S}=$ $\mathcal{S}(\Sigma)$ be the set of curves on $\Sigma$, that is, the set of finite unions of non-homotopic 
curves in $\mathcal{S}_{0}$. For simplicity we usually refer to elements of $\mathcal{S}$ as "curves" rather than "multicurves", in other words, a curve is not required to be connected. In addition by the term "loop" we mean "connected curve". The geometric intersection number $i(\alpha, \beta)$ between $\alpha, \beta \in \mathcal{S}$ is the least number of intersections between curves representing the two homotopy classes, that is,

$$
i(\alpha, \beta)=\min _{a \in \alpha, b \in \beta}|a \cap b| .
$$

Now given a surface $\Sigma=\Sigma_{g}^{b}$ of finite type and negative Euler characteristic, choose a maximal set $\mathcal{P C}=\left\{\sigma_{1}, \ldots, \sigma_{\xi}\right\}$ of homotopically distinct and non-boundary parallel loops in $\Sigma$ called pants curves, where $\xi=\xi(\Sigma)=3 g-3+b$ is the complexity of the surface. These connected curves split the surface into $k=2 g-2+b$ three-holed spheres $P_{1}, \ldots, P_{k}$, called pairs of pants. (Note that the boundary of $P_{i}$ may include punctures of $\Sigma$.) We refer to both the set $\mathcal{P}=\left\{P_{1}, \ldots, P_{k}\right\}$, and the set $\mathcal{P C}$, as a pants decomposition of $\Sigma$.

We take $P_{i}$ to be a closed three-holed sphere whose interior $\operatorname{Int}\left(P_{i}\right)$ is embedded in $\Sigma$; the closure of $\operatorname{Int}\left(P_{i}\right)$ fails to be embedded precisely in the case in which two of its boundary curves are identified in $\Sigma$, forming an embedded one-holed torus $\Sigma_{1,1}$. Thus each pants curve $\sigma=\sigma_{i}$ is the common boundary of one or two pants whose union we refer to as the modular surface associated to $\sigma$, denoted $M(\sigma)$. If the closure of $\operatorname{Int}\left(P_{i}\right)$ fails to be embedded then $M(\sigma)$ is a one-holed torus $\Sigma_{1,1}$, otherwise it is a four-holed sphere $\Sigma_{0,4}$.

Any hyperbolic pair of pants $P$ is made by gluing two right angled hexagons along three alternate edges which we call its seams. In much of what follows, it will be convenient to designate one of these hexagons as "white" and one as "black". A properly embedded arc in $P$, that is, an arc with its endpoints on $\partial P$, is called scc (same component connector) if it has both its endpoints on the same component of $\partial P$ and $d c c$ (different component connector) otherwise.

2.0.1 Convention on dual curves We shall need to consider dual curves to $\sigma_{i} \in \mathcal{P C}$, that is, curves which intersect $\sigma_{i}$ minimally and which are completely contained in $M\left(\sigma_{i}\right)$, the union of the pants $P, P^{\prime}$ adjacent to $\sigma_{i}$. The intersection number of such a connected curve with $\sigma_{i}$ is 1 if $M\left(\sigma_{i}\right)$ a one-holed torus and 2 otherwise. In the first case, the curve is made by identifying the endpoints of a single dcc-arc in the pair of pants adjacent to $\sigma_{i}$ and, in the second, it is the union of two scc-arcs, one in each of the two pants whose union is $M\left(\sigma_{i}\right)$. We adopt a useful convention introduced in [14] which simplifies the formulae in such a way as to avoid the need to distinguish between these two cases. Namely, for those $\sigma_{i}$ for which $M\left(\sigma_{i}\right)$ is $\Sigma_{1,1}$, we define the dual curve $D_{i} \in \mathcal{S}$ to be two parallel copies of the connected curve intersecting $\sigma_{i}$ 
once, while if $M\left(\sigma_{i}\right)$ is $\Sigma_{0,4}$ we take a single copy. In this way we always have, by definition, $i\left(\sigma_{i}, D_{i}\right)=2$, where $i(\alpha, \beta)$ is the geometric intersection number as above. A marking on $\Sigma$ is the specification of a fixed base surface $\Sigma_{0}$, together with a homeomorphism $\Psi: \Sigma_{0} \rightarrow \Sigma$. Markings can be defined in various equivalent ways, for example by specifying the choice of dual curves; see Section 3.1 below.

2.0.2 Convention on twists Our convention will always be to measure twists to the right as positive. We denote by $T w_{\sigma}(\gamma)$ the right Dehn twist of the curve $\gamma$ about the curve $\sigma$.

\section{Dehn-Thurston coordinates}

Suppose we are given a surface $\Sigma$ together with a pants decomposition $\mathcal{P}$ as above. Let $\gamma \in \mathcal{S}$ and for $i=1, \ldots, \xi$, let $q_{i}=i\left(\gamma, \sigma_{i}\right) \in \mathbb{Z}_{\geqslant 0}$. Notice that if $\sigma_{i_{1}}, \sigma_{i_{2}}, \sigma_{i_{3}}$ are pants curves which together bound a pair of pants whose interior is embedded in $\Sigma$, then the sum $q_{i_{1}}+q_{i_{2}}+q_{i_{3}}$ of the corresponding intersection numbers is even. The $q_{i}=q_{i}(\gamma)$ are sometimes called the length parameters of $\gamma$.

To define the twist parameter $\operatorname{tw}_{i}=\operatorname{tw}_{i}(\gamma) \in \mathbb{Z}$ of $\gamma$ about $\sigma_{i}$, we first have to fix a marking on $\Sigma$, for example by fixing a specific choice of dual curve $D_{i}$ to each pants curve $\sigma_{i}$; see Section 3.1 below. Then, after isotoping $\gamma$ into a well-defined standard position relative to $\mathcal{P}$ and to the marking, the twist $\mathrm{tw}_{i}$ is the signed number of times that $\gamma$ intersects a short arc transverse to $\sigma_{i}$. We make the convention that if $i\left(\gamma, \sigma_{i}\right)=0$, then $\operatorname{tw}_{i}(\gamma) \geqslant 0$ is the number of components in $\gamma$ freely homotopic to $\sigma_{i}$.

There are various ways of defining the standard position of $\gamma$, leading to differing definitions of the twist. The parameter $\operatorname{tw}_{i}(\gamma)=p_{i}(\gamma)$ which occurs in the statement of Theorem A is the one defined by Dylan Thurston [14], however in the proof of the formula we will find it convenient to use a slightly different definition $\operatorname{tw}_{i}(\gamma)=\hat{p}_{i}(\gamma)$ given by Penner [12]. Both of these definitions are explained in detail below, as is the precise relationship between them. With either definition, a classical theorem of Dehn [2] (see also Penner [12, page 12]) asserts that the length and twist parameters uniquely determine $\gamma$ :

Theorem 3.1 (Dehn's theorem) The map

$$
\Psi: \mathcal{S}(\Sigma) \rightarrow \mathbb{Z}_{\geqslant 0}^{\xi} \times \mathbb{Z}^{\xi}, \quad \gamma \in \mathcal{S}(\Sigma) \mapsto\left(q_{1}(\gamma), \ldots, q_{\xi}(\gamma) ; \operatorname{tw}_{1}(\gamma), \ldots, \operatorname{tw}_{\xi}(\gamma)\right)
$$

is an injection. The point $\left(q_{1}, \ldots, q_{\xi}, \mathrm{tw}_{1}, \ldots, \mathrm{tw}_{\xi}\right)$ is in the image of $\Psi$ (and hence corresponds to a curve) if and only if 
(i) if $q_{i}=0$, then $\mathrm{tw}_{i} \geqslant 0$, for each $i=1, \ldots, \xi$;

(ii) if $\sigma_{i_{1}}, \sigma_{i_{2}}, \sigma_{i_{3}}$ are pants curves which together bound a pair of pants whose interior is embedded in $\Sigma$, then the sum $q_{i_{1}}+q_{i_{2}}+q_{i_{3}}$ of the corresponding intersection numbers is even.

We remark that as a special case of (ii), the intersection number with a pants curve which bounds an embedded once-punctured torus or twice-punctured disk in $\Sigma$ is even.

One can think of this theorem in the following way. Suppose given a curve $\gamma \in \mathcal{S}$, whose length parameters $q_{i}(\gamma)$ necessarily satisfy the parity condition (ii), then the $q_{i}(\gamma)$ uniquely determine $\gamma \cap P_{j}$ for each pair of pants $P_{j}, j=1, \ldots, k$, in accordance with the possible arrangements of arcs in a pair of pants; see for example Penner [12]. Now given two pants adjacent along the curve $\sigma_{i}$, we have $q_{i}(\gamma)$ points of intersection coming from each side and we have only to decide how to match them together to recover $\gamma$. The matching takes place in the cyclic cover of an annular neighbourhood of $\sigma_{i}$. The twist parameter $\operatorname{tw}_{i}(\gamma)$ specifies which of the $\mathbb{Z}$ possible choices is used for the matching.

\subsection{The DT-twist}

In [14], Dylan Thurston gives a careful definition of the twist $\operatorname{tw}_{i}(\gamma)=p_{i}(\gamma)$ of $\gamma \in \mathcal{S}$ which is essentially the "folk" definition and the same as that implied in [3]. He observes that this definition has a nice intrinsic characterisation; see Section 3.1.4 below. Furthermore, it turns out to be the correct definition for our formula in Theorem A.

3.1.1 The marking Given the pants decomposition $\mathcal{P}$ of $\Sigma$, we note, following [14], that we can fix a marking on $\Sigma$ in three equivalent ways. These are

(a) a reversing map: an orientation-reversing map $R: \Sigma \rightarrow \Sigma$ so that for each $i=1, \ldots, \xi$ we have $R\left(\sigma_{i}\right)=\sigma_{i}$

(b) a hexagonal decomposition: this can be defined by a curve which meets each pants curve twice, decomposing each pair of pants into two hexagons;

(c) dual curves: for each $i$, a curve $D_{i}$ so that $i\left(D_{i}, \sigma_{j}\right)=2 \delta_{i j}$.

The characterisations (a) and (b) are most easily understood in connection with a particular choice of hyperbolic metric on $\Sigma$. Recall that a pair of pants $P$ is the union of two right-angled hexagons glued along its seams. There is an orientation reversing symmetry of $P$ which fixes the seams. The endpoints of exactly two seams meet each component of the boundary $\partial P$. Now let $\Sigma_{0}$ be a hyperbolic surface formed by gluing pants $P_{1}, \ldots, P_{k}$ in such a way that the seams are exactly matched on either side of 
each common boundary curve $\sigma_{i}$. In this case the existence of the orientation reversing map $R$ as in (a) and the hexagonal decomposition as in (b) are clear and are clearly equivalent.

If the modular surface associated to $\sigma_{i}$ is made up of two distinct pants $P, P^{\prime}$, then, as explained above, the dual curve $D_{i}$ to $\sigma_{i}$ is obtained by gluing the two scc-arcs in $P$ and in $P^{\prime}$ which run from $\sigma_{i}$ to itself. Each arc meets $\sigma_{i}$ orthogonally so that in the metric $\Sigma_{0}$ the two endpoints on each side of $\sigma_{i}$ are exactly matched by the gluing. If the modular surface is a single pair of pants $P$, then the dual curve is obtained by gluing the single dcc-arc in $P$ which runs from $\sigma_{i}$ to itself. Once again both ends of this arc meet $\sigma_{i}$ orthogonally and in the metric $\Sigma_{0}$ are exactly matched by the gluing. In this case, following the convention explained in Section 2, we take the dual curve $D_{i}$ to be two parallel copies of the loop just described. Thus in all cases $i\left(D_{i}, \sigma_{j}\right)=2 \delta_{i j}$ and furthermore the curves $D_{i}$ are fixed by $R$.

A general surface $\Sigma$ can be obtained from $\Sigma_{0}$ by performing a Fenchel-Nielsen twist about each $\sigma_{i}$. Namely, if $A_{i}=\sigma_{i} \times[0,1]$ is an annulus around $\sigma_{i}$ and if we parameterise $\sigma_{i}$ as $s \mapsto \sigma_{i}(s) \in \Sigma$ for $s \in[0,1)$, then the distance $t$ twist, denoted $\mathrm{FN}_{t}: \Sigma_{0} \rightarrow \Sigma$, maps $A_{i}$ to itself by $\left(\sigma_{i}(s), \theta\right) \mapsto\left(\sigma_{i}(s+\theta t), \theta\right)$ and is the identity elsewhere. Clearly $\mathrm{FN}_{t}$ induces a reversing map, a hexagonal decomposition, and dual curves on the surface $\mathrm{FN}_{t}(\Sigma)$, showing that each of (a), (b) and (c) equivalently define a marking on an arbitrary surface $\Sigma$.

3.1.2 The twist Having defined the marking, we can now define the twist $p_{i}(\gamma)$ for any $\gamma \in \mathcal{S}$. Arrange, as above, the dual curves $D_{i}$ to be fixed by $R$, so that, in particular, if $\sigma_{i}$ is the boundary of a single pair of pants $P$, then the two parallel components of the curve $D_{i}$ are contained one in each of the two hexagons making up $P$. For each $i=1, \ldots, \xi$, choose a small annular neighbourhood $A_{i}$ of $\sigma_{i}$, in such a way that the complement $\Sigma \backslash \bigcup_{i=1}^{\xi} \operatorname{Int}\left(A_{i}\right)$ of the interiors of these annuli in $\Sigma$ are pants $\widehat{P}_{1}, \ldots, \widehat{P}_{k}$. Arrange $\gamma$ so that its intersection with each $\widehat{P}_{i}$ is fixed by $R$ and so that it is transverse to $D_{i}$. Also push any component of $\gamma$ parallel to any $\sigma_{i}$ into $A_{i}$.

If $q_{i}=i\left(\gamma, \sigma_{i}\right)=0$, define $p_{i} \geq 0$ to be the number of components of $\gamma$ parallel to $\sigma_{i}$. Otherwise, $q_{i}=i\left(\gamma, \sigma_{i}\right)>0$. In this case, orient both $\gamma \cap A_{i}$ and $D_{i} \cap A_{i}$ to run consistently from one boundary component of $A_{i}$ to the other. (If $M\left(\sigma_{i}\right)$ is $\Sigma_{0,4}$, then the two arcs of $D_{i} \cap A_{i}$ will be oriented in opposite directions relative to the connected curve $D_{i}$.) Then define

$$
p_{i}=\hat{i}\left(\gamma \cap A_{i}, D_{i} \cap A_{i}\right),
$$

where $\hat{i}(\alpha, \beta)$ is the algebraic intersection number between the curves $\alpha$ and $\beta$, namely the sum of the indices of the intersection points of $\alpha$ and $\beta$, where an intersection 
point is of index +1 when the orientation of the intersection agrees with the orientation of $\Sigma$ and -1 otherwise.

Note that this definition is independent of both the choice of the orientations of $\gamma \cap A_{i}$ and $D_{i} \cap A_{i}$, and of the choice of the arrangement of $\gamma$ in the pants adjacent to $\sigma_{i}$. Also note that, following the convention about dual curves in Section 2.0.1, $p_{i}$ is always even. Two simple examples are illustrated in Figures 1 and 2.

3.1.3 An alternative definition The twist $p_{i}$ can also be described in a slightly different way as follows. Lift $A_{i}$ to its $\mathbb{Z}$-cover which is an infinite strip $H$. As shown in Figures 1 and 2, the lifts of $D_{i} \cap A_{i}$ are arcs joining the two boundaries $\partial_{0} H$ and $\partial_{1} H$ of $H$. They are equally spaced like rungs of a ladder in such a way that there are exactly two lifts in any period of the translation corresponding to $\sigma_{i}$. Any arc of $\gamma$ enters $H$ on one side and leaves on the other. Fix such a rung $D_{*}$ say and number the strands of $\gamma$ meeting $\partial_{0} H$ in order as $X_{n}, n \in \mathbb{Z}$, where $X_{0}$ is the first arc to the right of $D_{*}$ and $n$ increases moving to the right along $\partial_{0} H$, relative to the orientation of the incoming strand of $\gamma$. Label the endpoints of $\gamma$ on $\partial_{1} H$ by $X_{n}^{\prime}, n \in \mathbb{Z}$ correspondingly, as shown in Figure 1. Since $\gamma$ is simple, if $X_{0}$ is matched to $X_{r}^{\prime}$, then $X_{n}$ is matched to $X_{n+r}^{\prime}$ for all $n \in \mathbb{Z}$. Then it is not hard to see that $r=p_{i} / 2$.

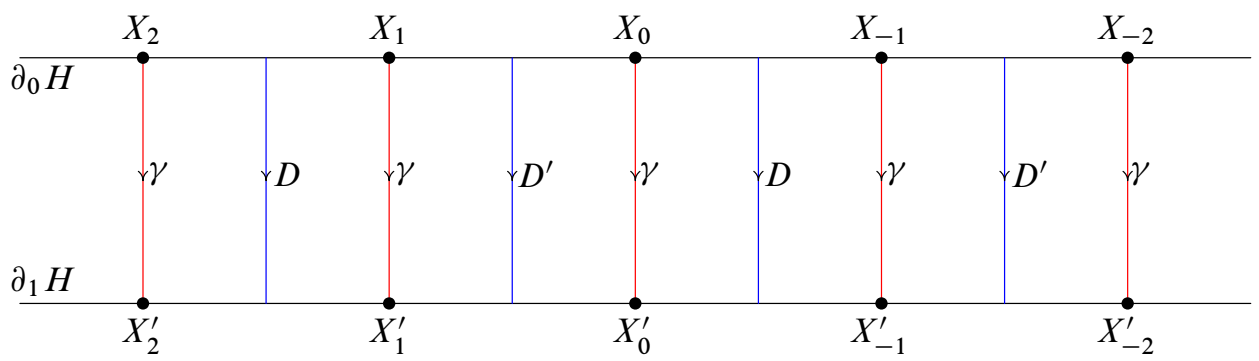

Figure 1: A curve $\gamma$ with $p_{i}(\gamma)=0$. The $\operatorname{arcs} D, D^{\prime}$ together project to the dual curve $D_{i}$.

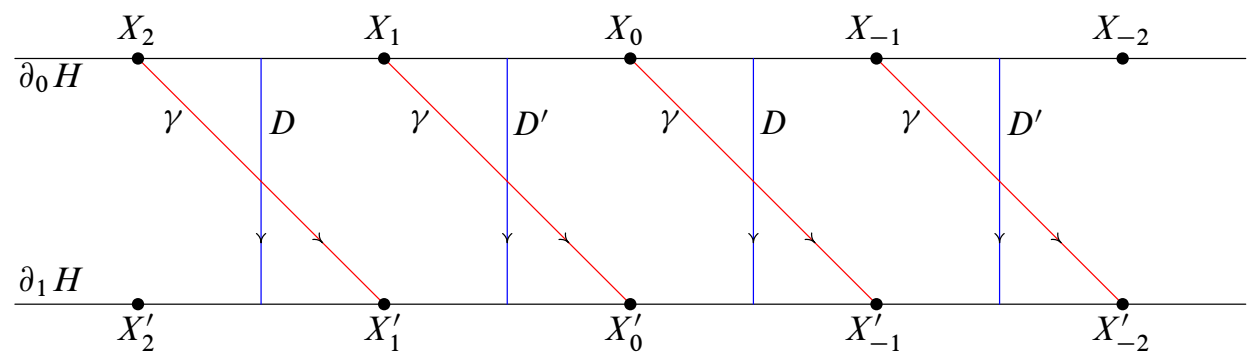

Figure 2: A curve $\gamma$ with $p_{i}(\gamma)=-2$ 
3.1.4 Intrinsic characterisation The intrinsic characterisation of the twist in [14] uses the Luo product $\alpha \cdot \beta$ of curves $\alpha, \beta \in \mathcal{S}$ on an oriented surface $\Sigma$. This is defined as follows [6;14]:

- If $a \cap b=\varnothing$, then $\alpha \cdot \beta=\alpha \cup \beta \in \mathcal{S}$.

- Otherwise, arrange $\alpha$ and $\beta$ in minimal position, that is, such that $i(\alpha \cap \beta)=$ $|\alpha \cap \beta|$. In a neighbourhood of each intersection point $x_{j} \in \alpha \cap \beta$, replace $\alpha \cup \beta$ by the union of the two arcs which turn left from $\alpha$ to $\beta$ relative to the orientation of $\Sigma$; see Figure 3. (In [6] this is called the resolution of $\alpha \cup \beta$ from $\alpha$ to $\beta$ at $x_{j}$.) Then $\alpha \cdot \beta$ is the curve made up from $\alpha \cup \beta$ away from the points $x_{j}$, and the replacement arcs near each $x_{j}$.

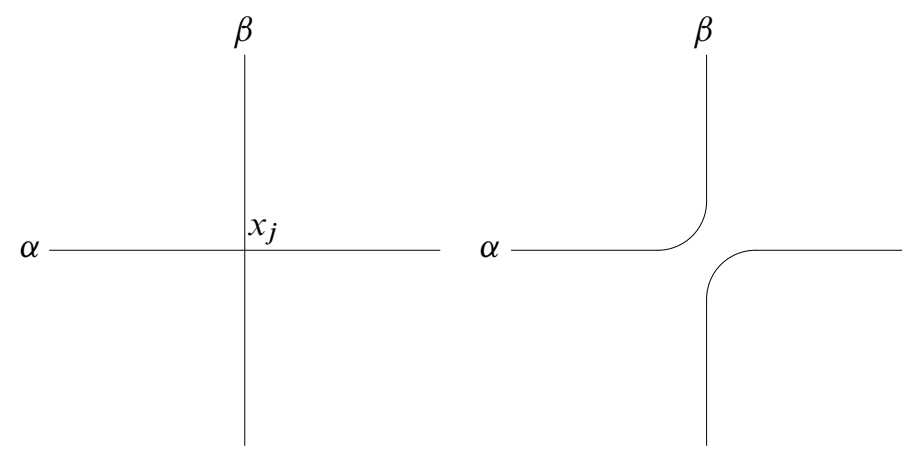

Figure 3: The Luo product: the resolution of $\alpha \cup \beta$ at $x_{j}$

Proposition 3.2 [14, Definition 15] The function $p_{i}: \mathcal{S}(\Sigma) \rightarrow \mathbb{Z}$ is the unique function such that for all $\gamma \in \mathcal{S}$,

(i) $p_{i}\left(\sigma_{j} \cdot \gamma\right)=p_{i}(\gamma)+2 \delta_{i j}$;

(ii) $p_{i}$ depends only on the restriction of $\gamma$ to the pants adjacent to $\sigma_{i}$;

(iii) $p_{i}(R(\gamma))=-p_{i}(\gamma)$, where $R$ is the orientation reversing involution of $\Sigma$ defined above.

We call $p_{i}(\gamma)$ the DT-twist parameter of $\gamma$ about $\sigma_{i}$. Property (i) fixes our convention noted above that the right twist is taken positive. Notice that $p_{i}\left(D_{i}\right)=0$. We also observe:

Proposition 3.3 Let $\gamma \in \mathcal{S}$. Then

$$
p_{i}\left(T w_{\sigma_{i}}(\gamma)\right)=p_{i}(\gamma)+2 q_{i} .
$$


3.1.5 Relation to [3] In [3], a curve $\gamma \in \mathcal{S}$ is parameterized by three non-negative integers $\left(m_{i}, s_{i}, t_{i}\right)$. These are defined as the intersection numbers of $\gamma$ with the three curves $K_{i}, K_{i}^{\prime}$ and $K_{i}^{\prime \prime}$, namely the pants curve $\sigma_{i}$, its dual curve $D_{i}$, and $T w_{\sigma_{i}}\left(D_{i}\right)$, the right Dehn twist of $D_{i}$ about $\sigma_{i}$; see Figure 4 on page 62 in [3]. In particular,

- $m_{i}(\gamma)=i\left(\gamma, K_{i}\right)=i\left(\gamma, \sigma_{i}\right)=q_{i}(\gamma)$;

- $s_{i}(\gamma)=i\left(\gamma, K_{i}^{\prime}\right)=i\left(\gamma, D_{i}\right)=\left|p_{i}(\gamma)\right| / 2$;

- $t_{i}(\gamma)=i\left(\gamma, K_{i}^{\prime \prime}\right)=i\left(\gamma, T w_{\sigma_{i}}\left(D_{i}\right)\right)=\left|p_{i}(\gamma) / 2-q_{i}(\gamma)\right|$.

As proved in [3], the three numbers $m_{i}, s_{i}$ and $t_{i}$ satisfy one of the three relations $m_{i}=s_{i}+t_{i} ; s_{i}=m_{i}+t_{i} ; t_{i}=m_{i}+s_{i}$. As it is easily verified by a case-by-case analysis, we have:

Theorem 3.4 Each triple $\left(m_{i}, s_{i}, t_{i}\right)$ uniquely determines and is determined by the parameters $q_{i}$ and $p_{i}$. In fact, $q_{i}=m_{i}$ and $p_{i}=2 \operatorname{sign}\left(p_{i}\right) s_{i}$ where

$$
\operatorname{sign}\left(p_{i}\right)= \begin{cases}+1 & \text { if } m_{i}=s_{i}+t_{i} \text { or } s_{i}=m_{i}+t_{i}, \\ -1 & \text { if } t_{i}=m_{i}+s_{i} .\end{cases}
$$

Proof If $\operatorname{sign}\left(p_{i}\right)=-1$, then $p_{i} / 2-q_{i} \leq 0$. So

$$
t_{i}=\left|\frac{p_{i}}{2}-q_{i}\right|=-\left(\frac{p_{i}}{2}-q_{i}\right)=\frac{\left|p_{i}\right|}{2}+q_{i}=s_{i}+m_{i} .
$$

If $\operatorname{sign}\left(p_{i}\right)=+1$, then

$$
\begin{aligned}
& \text { if } \frac{p_{i}}{2} \leqslant q_{i}, \text { then } t_{i}=\left|\frac{p_{i}}{2}-q_{i}\right|=q_{i}-\frac{\left|p_{i}\right|}{2}=m_{i}-s_{i}, \\
& \text { if } q_{i} \leqslant \frac{p_{i}}{2}, \text { then } t_{i}=\left|\frac{p_{i}}{2}-q_{i}\right|=\frac{\left|p_{i}\right|}{2}-q_{i}=s_{i}-m_{i},
\end{aligned}
$$

as we wanted to prove.

\subsection{The P-twist}

We now summarise Penner's definition of the twist parameter following [12, Section 1.2]. Instead of arranging the arcs of $\gamma$ transverse to $\sigma_{i}$ symmetrically with respect to the involution $R$, we now arrange them to cross $\sigma_{i}$ through a short closed arc $w_{i} \subset \sigma_{i}$. There is some choice to be made in how we do this, which leads to the difference with the definition of the previous section. It is convenient to think of $w_{i}$ as contained in the two "front" hexagons of the pants $P$ and $P^{\prime}$ glued along $\sigma_{i}$, which we will also refer to as the "white" hexagons. 
Precisely, for each pants curve $\sigma_{i} \in \mathcal{P C}$, fix a short closed arc $w_{i} \subset \sigma_{i}$, which we take to be symmetrically placed in the white hexagon of one of the adjacent pants $P$, midway between the two seams which meet $\sigma_{i} \subset \partial P$. For each $\sigma_{i}$, fix an annular neighbourhood $A_{i}$ and extend $w_{i}$ into a "rectangle" $R_{i} \subset A_{i}$ with one edge on each component of $\partial A_{i}$ and "parallel" to $w_{i}$ and two edges arcs from one component of $\partial A_{i}$ to the other. (See Penner [12] for precise details.)

Now isotope $\gamma \in \mathcal{S}$ into Penner standard position as follows. Any component of $\gamma$ homotopic to $\sigma_{i}$ is isotoped into $A_{i}$. Next, arrange $\gamma$ so that it intersects each $\sigma_{i}$ exactly $q_{i}(\gamma)$ times and moreover so that all points in $\gamma \cap \sigma_{i}$ are contained in $w_{i}$. We further arrange that all the twisting of $\gamma$ occurs in $A_{i}$. Precisely, isotope so that $\gamma \cap \partial A_{i} \subset \partial R_{i}$, in other words, so that $\gamma$ enters $A_{i}$ across the edges of $R_{i}$ parallel to $w_{i}$. By pushing all the twisting into $A_{i}$, we can also arrange that outside $A_{i}$, any dcc-arc of $\gamma \cap P$ does not cross any seam of $P$. The scc-arcs are slightly more complicated. Any such arc has both endpoints on the same boundary component, let us say $\partial_{0} P$. Give the white hexagon (the "front" hexagon in Figure 4) the same orientation as the surface $\Sigma$. With this orientation, the two other boundary components $\partial_{1} P$ and $\partial_{\infty} P$ are arranged as shown in the figure. We isotope the scc-arc so that outside $A_{i}$ it loops round the right hand component $\partial_{1} P$, cutting the seam which is to the right of the seam contained in $\partial_{0} P$ exactly; see Figure 4.

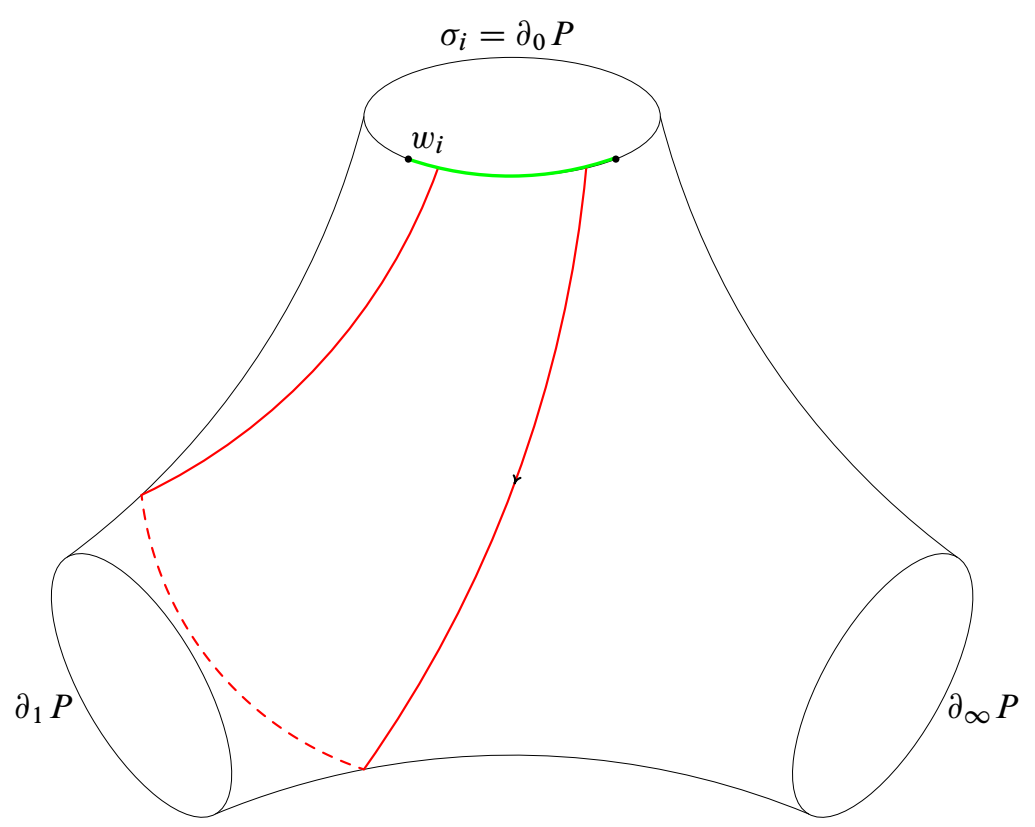

Figure 4: An scc-arc in Penner standard position 
Having put $\gamma$ into Penner standard position, we define the Penner-twist or $P$-twist $\hat{p}_{i}(\gamma)$ as follows. Let $d_{i}$ be a short arc transverse to $w_{i}$ with one endpoint on each of the two components of $\partial A_{i}$.

- If $q_{i}(\gamma)=i\left(\gamma, \sigma_{i}\right)=0$, let $\hat{p}_{i}(\gamma) \geqslant 0$ be the number of components of $\gamma$ which are freely homotopic to $\sigma_{i}$.

- If $q_{i}(\gamma) \neq 0$, let $\left|\hat{p}_{i}(\gamma)\right|$ be the minimum number of arcs of $\gamma \cap A_{i}$ which intersect $d_{i}$, where the minimum is over all families of arcs properly embedded in $A_{i}$, isotopic to $\gamma \cap A_{i}$ by isotopies fixing $\partial A$ pointwise. Take $\hat{p}_{i}(\gamma) \geqslant 0$ if some components of $\gamma$ twist to the right in $A_{i}$ and $\hat{p}_{i}(\gamma) \leqslant 0$ otherwise. (There cannot be components twisting in both directions since $\gamma$ is embedded and, if there is no twisting, then $\hat{p}_{i}(\gamma)=0$.)

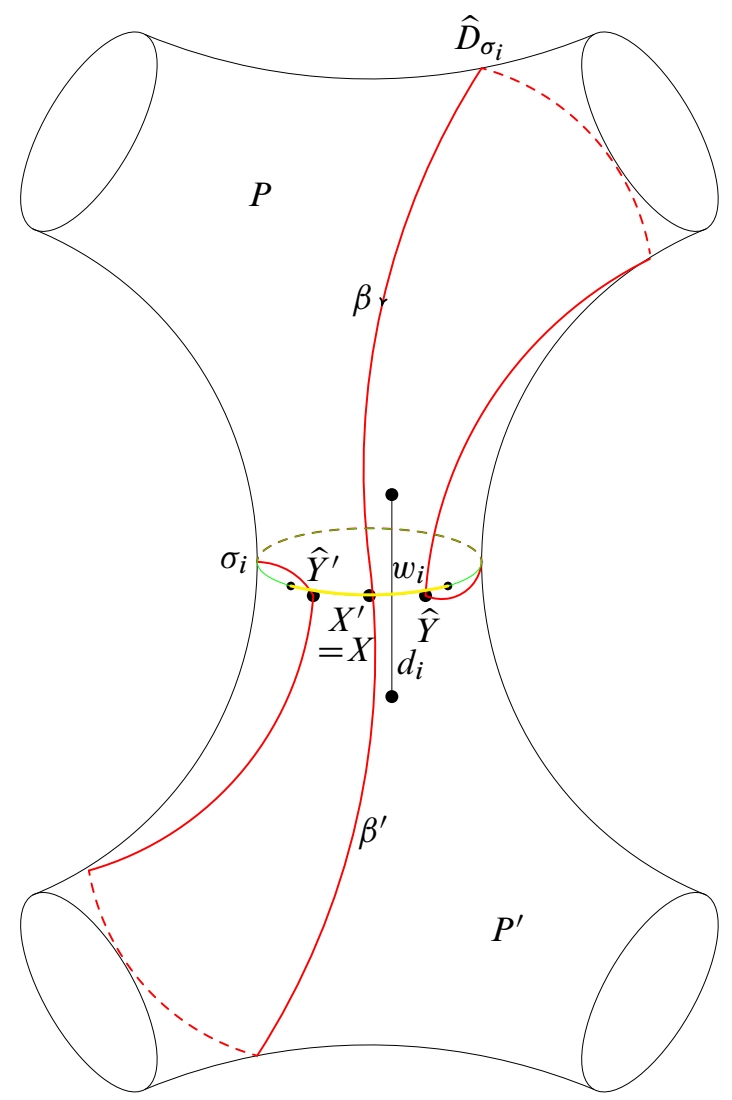

Figure 5: The dual curve $D_{i}$ in Penner standard position. The endpoints of $d_{i}$ are on the annulus $A_{i}$ (not shown) around $\sigma_{i}$. 
3.2.1 The dual curves in Penner position As an example, we explain how to put the dual curves $D_{i}$ into Penner standard position. This requires some care. For clarity, we denote one component of the dual curve $D_{i}$ by $\hat{D}_{i}$, so that in the case in which $M\left(\sigma_{i}\right)$ is $\Sigma_{1,1}$, we have $2 \widehat{D}_{i}=D_{i}$, while $\widehat{D}_{i}=D_{i}$ otherwise.

If $M\left(\sigma_{i}\right)$ is $\Sigma_{1,1}$, there is only one arc to be glued whose endpoints we can arrange to be in $w_{i}$. We simply take two parallel copies of this loop $\widehat{D}_{i}$ so that $D_{i}=2 \widehat{D}_{i}$ and $\hat{p}_{i}\left(D_{i}\right)=0$.

If $M\left(\sigma_{i}\right)$ is $\Sigma_{0,4}$ then $D_{i}=\widehat{D}_{i}$. In this case we have to match the endpoints of two scc-arcs $\beta \subset P$ and $\beta^{\prime} \subset P^{\prime}$, both of which have endpoints on $\sigma_{i}$. The arc $\beta$ has one endpoint $X$ in the front white hexagon of $P$, which we can arrange to be in $w_{i}$, and the other $Y$ in the symmetrical position in the black hexagon. Label the endpoints of $\beta^{\prime}$ in a similar way. To get $\beta \cup \beta^{\prime}$ into standard Penner position, we have to move the back endpoints $Y$ and $Y^{\prime}$ round to the front so that they also lie in $w_{i}$. Arrange $P$ and $P^{\prime}$ as shown in Figure 5 with the white hexagons to the front. In Penner position, $\beta$ has to loop round the right hand boundary component of $P$ so that $Y$ has to move to a point $\hat{Y}$ to the right of $X$ along $w_{i}$ in Figure 5. In $P^{\prime}$ on the other hand, $\beta^{\prime}$ has to loop round the right hand boundary component of $P^{\prime}$, so that $Y^{\prime}$ gets moved to a point $\hat{Y}^{\prime}$ to the left of $X^{\prime}$ on $w_{i}$. Since $X$ is identified to $X^{\prime}$, to avoid self-intersections, $\hat{Y}$ has to be joined to $\hat{Y}^{\prime}$ by a curve which follows $\sigma_{i}$ around the back of $P \cup P^{\prime}$. By inspection, we see that $\hat{p}_{i}\left(\hat{D}_{i}\right)=-1$.

\subsection{Relationship between the different definitions of twist}

Our proof of Theorem A in Section 6 uses the explicit relationship between the above two definitions of the twist. The formula in Theorem 3.5 below appears without proof in [14]; for completeness we supply a proof.

Suppose that two pairs of pants meet along $\sigma_{i} \in \mathcal{P C}$. Label their respective boundary curves $(A, B, E)$ and $(C, D, E)$ in clockwise order, where $E=\sigma_{i}$; see Figure 6. (Some of these boundary curves may be identified in $\Sigma$.)

Theorem 3.5 [14, Appendix B] As above, let $\gamma \in \mathcal{S}$ and let $q_{i}=q_{i}(\gamma), \hat{p}_{i}=\hat{p}_{i}(\gamma)$ and $p_{i}=p_{i}(\gamma)$ respectively denote its length parameter, its $P$-twist and its DT-twist around $\sigma_{i}$. Then

$$
\hat{p}_{i}=\frac{p_{i}+l(A, E ; B)+l(C, E ; D)-q_{i}}{2},
$$

where $l(X, Y ; Z)$ denotes the number of strands of $\gamma \cap P$ running from the boundary curve $X$ to the boundary curve $Y$ in the pair of pants $P$ having boundary curves $(X, Y, Z)$. 


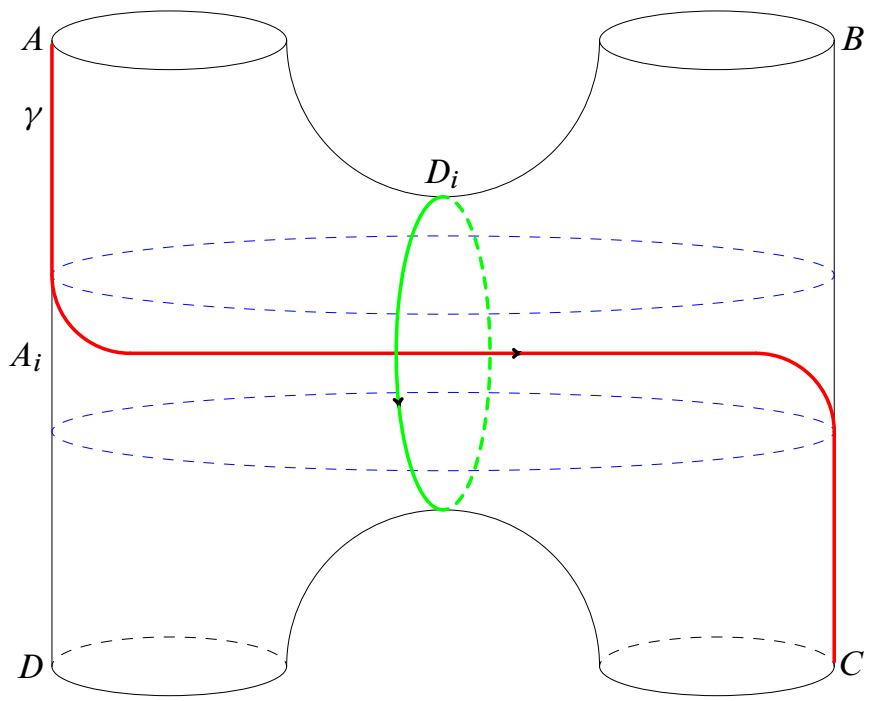

Figure 6: Case $n=1$ when $\gamma$ goes from $A$ to $C . E$ is the core curve of the annulus $A_{i}$.

Proof Let $\gamma \in \mathcal{S}$. We will use a case-by-case analysis to give a proof by induction on $n=q_{i}(\gamma)$. We shall assume that the modular surface $M\left(\sigma_{i}\right)$ is $\Sigma_{0,4}$, so that $\sigma_{i}$ belongs to the boundary of two distinct pants $P=(A, B, E)$ and $P^{\prime}=(C, D, E)$, and leave the case in which $M\left(\sigma_{i}\right)$ is $\Sigma_{1,1}$ to the reader. We begin with the cases $n=1$ and $n=2$, because $n=2$ is useful for the inductive step.

When $n=1$, the strand of $\gamma$ which intersects $\sigma_{i}$ must join one of the boundary components of $P$ different from $E$, to one of the two boundary components of $P^{\prime}$ different from $E$. We have four cases corresponding to $\gamma$ joining $A$ or $B$ to $C$ or $D$. Figure 6 shows the case in which $\gamma$ joins $A$ to $C$. One checks easily that $l(A, E ; B)=1, l(C, E ; D)=1$ while $\hat{p}=0, p=-1$ and $q=1$, verifying the formula in this case. The other cases are similar.

Now consider $n=2$, so that $\gamma \cap M\left(\sigma_{i}\right)$ may have either one or two connected components. If there are two components, then each one was already analysed in the case $n=1$, and the result follows by the additivity of the quantities involved.

If $\gamma \cap P$ is connected, we must have (in one of the pants $P$ or $P^{\prime}$ ) an scc-arc which has both its endpoints on $\sigma_{i}$. Without loss of generality we may suppose that this arc is in $P$. Choose an orientation on $\gamma$ and call its initial and final points $X_{1}$ and $X_{2}$ respectively. The endpoints of this arc must be joined to the boundary components $C$ or $D$ of $P^{\prime}$. Figure 7 illustrates the case in which $X_{1}$ is joined to $D$, while $X_{2}$ is 


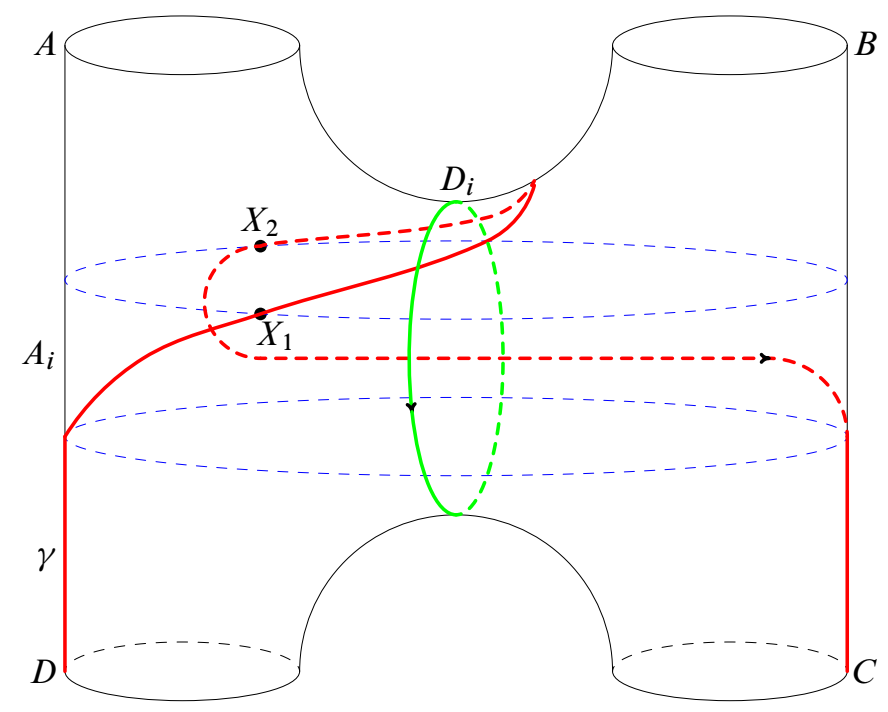

Figure 7: Case $n=2$ when $X_{1}$ is joined to $D$ and $X_{2}$ is joined to $C$

joined to $C$. Then $l(A, E ; B)=0, l(C, E ; D)=1$ while $\hat{p}=0, p=1$ and $q=2$, verifying the formula in this case. The other cases are similar.

Suppose now that the statement is true for any $n<q_{i}$. If $\gamma \cap M\left(\sigma_{i}\right)$ is not connected, then each connected component intersects $\sigma_{i}$ less then $n$ times and the result follows from the inductive hypothesis and the additivity of the quantities involved.

If $\hat{\gamma}=\gamma \cap M\left(\sigma_{i}\right)$ is connected, then there will be an arc which has both its endpoints on $\sigma_{i}$. Choose an orientation on $\gamma$. Without loss of generality, we can suppose that the first such arc is contained in $P$. Let $X_{1}$ and $X_{2}$ be its two ordered endpoints. Then $X_{2}$ splits $\hat{\gamma}$ into two oriented curves $\alpha$ and $\beta$, where $\alpha$ contains only one arc with both endpoints in $\sigma_{i}$, while $\beta$ has $n-1$ arcs of this kind. Now we modify $\alpha$ and $\beta$, in such a way that they will became properly embedded arcs in $M\left(\sigma_{i}\right)$, that is, arcs with endpoints on $\partial\left(M\left(\sigma_{i}\right)\right) \subset \Sigma$. We do this by adding a segment for each one of $\alpha$ and $\beta$ from $X_{2}$ to one of the boundary components $C$ or $D$ of $P^{\prime}$. In order to respect the orientation of $\alpha$ and $\beta$ we add the segment twice, once with each orientation. This will not change the quantities involved. For example, suppose we add two segments from $X_{2}$ to $C$. This creates two oriented curves $\alpha^{\prime}$ and $\beta^{\prime}$ in $M\left(\sigma_{i}\right)$ such that

$$
t_{i}(\gamma)=t_{i}(\alpha \cup \beta)=t_{i}\left(\alpha^{\prime} \cup \beta^{\prime}\right)=t_{i}\left(\alpha^{\prime}\right)+t_{i}\left(\beta^{\prime}\right)
$$

and the conclusion now follows from the inductive hypothesis. 
Remark 3.6 There is a nice formula for the number $l(X, Y, Z)$ in the above theorem. Given $a, b \in \mathbb{R}$, let $\max (a, b)=a \vee b$ and $\min (a, b)=a \wedge b$. Suppose that a pair of pants has boundary curves $X, Y, Z$ and that $\gamma \in \mathcal{S}$. Let $x=i(\gamma, X)$ and define $y, z$ similarly. As above let $l(X, Y ; Z)$ denote the number of strands of $\gamma$ running from $X$ to $Y$. Then (see Thurston [14, page 20])

$$
l(X, Y ; Z)=0 \vee\left(\frac{x+y-z}{2} \wedge x \wedge y\right) .
$$

\section{The gluing construction}

As explained in the introduction, the representations which we shall consider are holonomy representations of projective structures on $\Sigma$, chosen so that the holonomies of all the loops $\sigma_{j} \in \mathcal{P C}$ determining the pants decomposition $\mathcal{P}$ are parabolic. The interior of the set of free, discrete, and geometrically finite representations of this form is called the Maskit embedding of $\Sigma$; see Section 4.5 below.

The construction of the projective structure on $\Sigma$ is based on Kra's plumbing construction [5]; see Section 4.4. However it will be convenient to describe it in a somewhat different way. The idea is to manufacture $\Sigma$ by gluing triply punctured spheres across punctures. There is one triply punctured sphere for each pair of pants $P \in \mathcal{P}$, and the gluing across the pants curve $\sigma_{j}$ is implemented by a specific projective map depending on a parameter $\tau_{j} \in \mathbb{C}$. The $\tau_{j}$ will be the parameters of the resulting holonomy representation $\rho: \pi_{1}(\Sigma) \rightarrow \operatorname{PSL}(2, \mathbb{C})$.

More precisely, we first fix an identification of the interior of each pair of pants $P_{i}$ to a standard triply punctured sphere $\mathbb{P}$. We endow $\mathbb{P}$ with the projective structure coming from the unique hyperbolic metric on a triply punctured sphere. The gluing is carried out by deleting open punctured disk neighbourhoods of the two punctures in question and gluing horocyclic annular collars round the resulting two boundary curves; see Figure 8.

\subsection{The gluing}

To describe the gluing in detail, first recall (see eg Mumford, Series and Wright [11, page 207]) that any triply punctured sphere is isometric to the standard triply punctured sphere $\mathbb{P}=\mathbb{H} / \Gamma$, where

$$
\Gamma=\left\langle\left(\begin{array}{ll}
1 & 2 \\
0 & 1
\end{array}\right),\left(\begin{array}{ll}
1 & 0 \\
2 & 1
\end{array}\right)\right\rangle .
$$



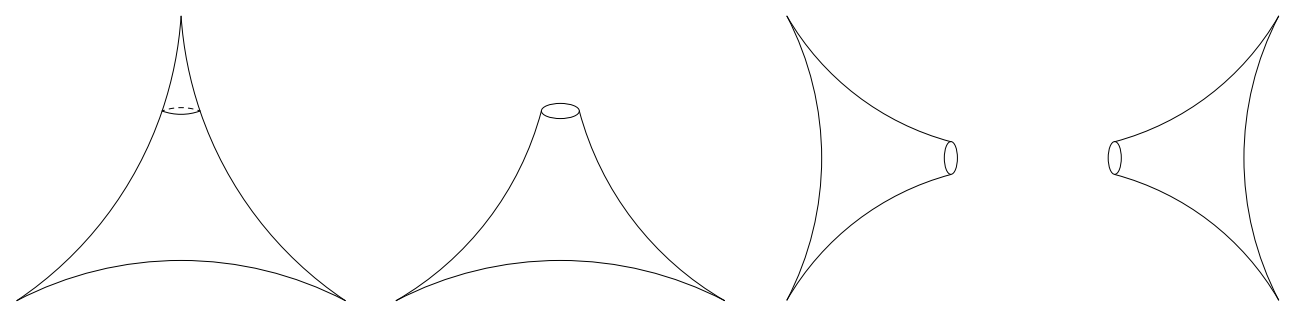

Figure 8: Deleting horocyclic neighbourhoods of the punctures and preparing to glue

Fix a standard fundamental domain for $\Gamma$ as shown in Figure 9, so that the three punctures of $\mathbb{P}$ are naturally labelled $0,1, \infty$. Let $\Delta_{0}$ be the ideal triangle with vertices $\{0,1, \infty\}$, and $\Delta_{1}$ be its reflection in the imaginary axis. We sometimes refer to $\Delta_{0}$ as the white triangle and $\Delta_{1}$ as the black.

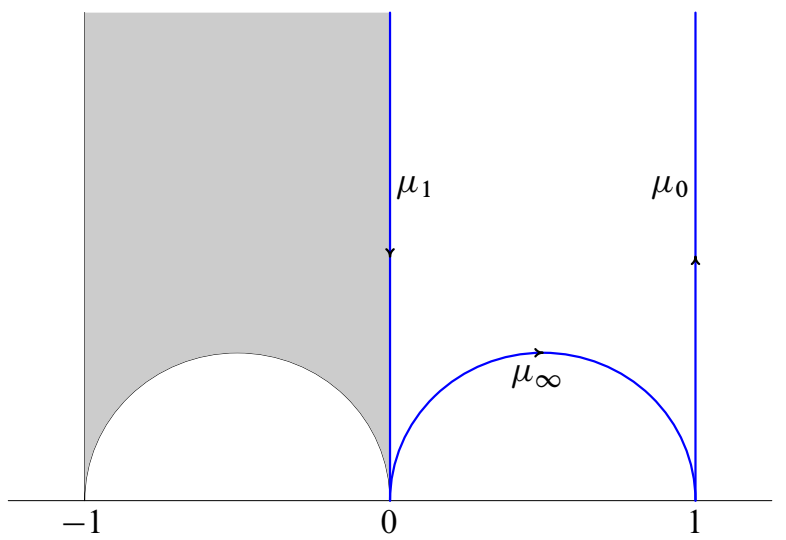

Figure 9: The standard fundamental domain for $\Gamma$. The white triangle $\Delta_{0}$ is unshaded.

With our usual pants decomposition $\mathcal{P}$, fix homeomorphisms $\Phi_{i}$ from the interior of each pair of pants $P_{i}$ to $\mathbb{P}$. This identification induces a labelling of the three boundary components of $P_{i}$ as $0,1, \infty$ in some order, fixed from now on. We denote the boundary labelled $\epsilon \in\{0,1, \infty\}$ by $\partial_{\epsilon} P_{i}$. The identification also induces a colouring of the two right angled hexagons whose union is $P_{i}$, one being white and one being black. Suppose that the pants $P, P^{\prime} \in \mathcal{P}$ are adjacent along the pants curve $\sigma$ meeting along boundaries $\partial_{\epsilon} P$ and $\partial_{\epsilon^{\prime}} P^{\prime}$. (If $P=P^{\prime}$ then clearly $\epsilon \neq \epsilon^{\prime}$.) The gluing across $\sigma$ will be described by a complex parameter $\tau$ with $\Im \tau>0$, called the plumbing parameter of the gluing. We first describe the gluing in the case $\epsilon=\epsilon^{\prime}=\infty$.

Arrange the pants with $P$ on the left as shown in Figure 10. (Note that the illustration in the figure describes the more general case $\epsilon=1$ and $\epsilon^{\prime}=0$.) Take two copies $\mathbb{P}, \mathbb{P}^{\prime}$ 

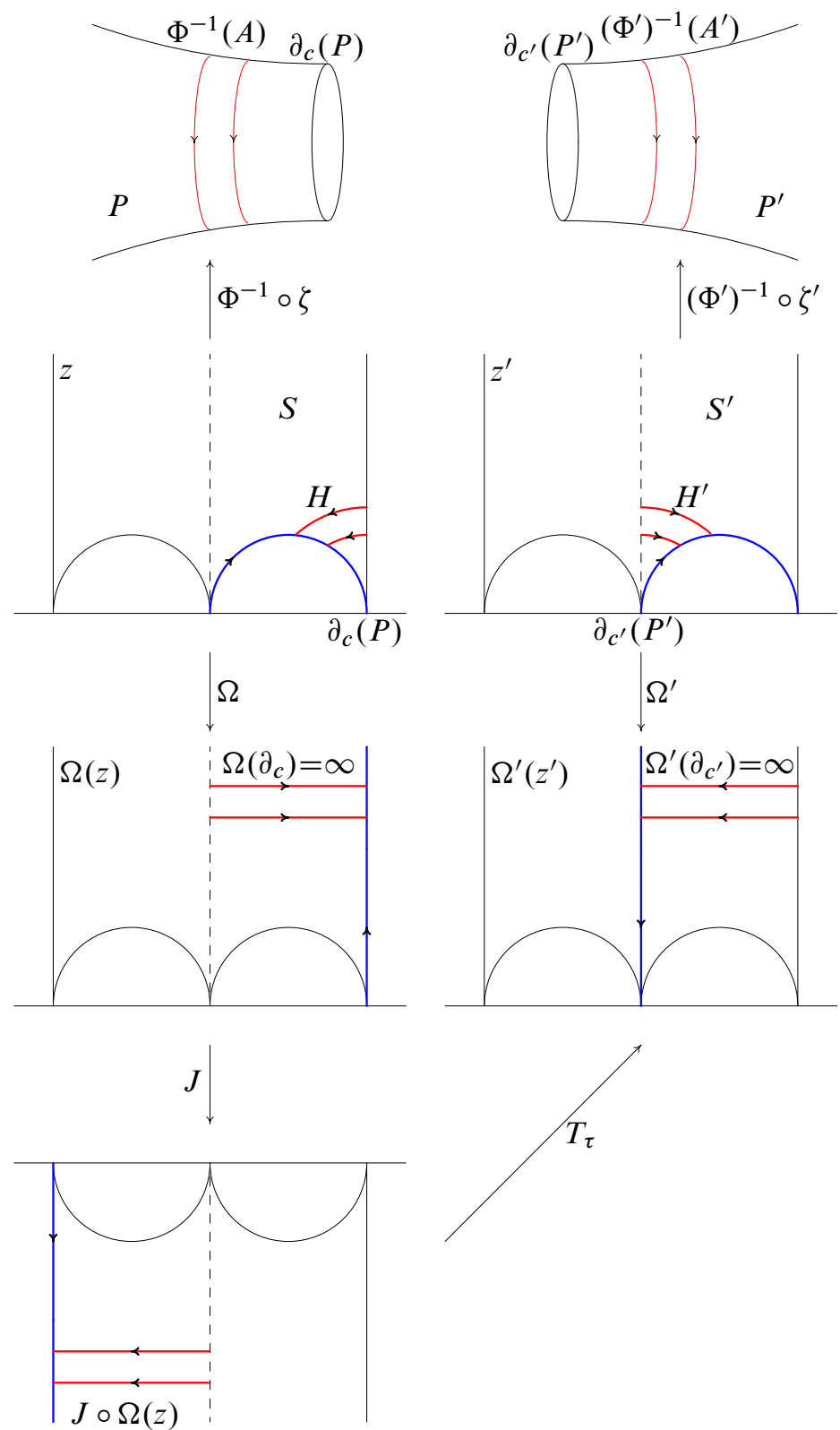

Figure 10: The gluing construction when $\epsilon=1$ and $\epsilon^{\prime}=0$. The top two upwards pointing arrows are the restrictions of $\Phi^{-1} \circ \xi$ and $\left(\Phi^{\prime}\right)^{-1} \circ \xi^{\prime}$ to the fundamental region $\Delta_{0} \cup \Delta_{1}$. The strips $H=H_{1}, H^{\prime}=H_{0}^{\prime}$ project under $\zeta, \zeta^{\prime}$ to the annuli $A \subset \mathbb{P}, A^{\prime} \subset \mathbb{P}^{\prime}$ whose core curves are $h_{0}, h_{1}^{\prime}$; these are identified with the corresponding annuli and curves on $P, P^{\prime}$ by $\Phi^{-1}, \Phi^{\prime-1}$. Only the parts of $H, H^{\prime}$ in $\Delta_{0}$ are shown. 
of $\mathbb{P}$. Each of these is identified with $\mathbb{H} / \Gamma$ as described above. We refer to the copy of $\mathbb{H}$ associated to $\mathbb{P}^{\prime}$ as $\mathbb{H}^{\prime}$ and denote the natural parameters in $\mathbb{H}, \mathbb{H}^{\prime}$ by $z, z^{\prime}$ respectively. Let $\zeta$ and $\zeta^{\prime}$ be the projections $\zeta: \mathbb{H} \rightarrow \mathbb{P}$ and $\zeta^{\prime}: \mathbb{H}^{\prime} \rightarrow \mathbb{P}^{\prime}$ respectively.

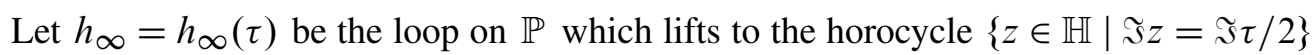
on $\mathbb{H}$. For $\eta>0, H_{\infty}=H_{\infty}(\tau, \eta)=\{z \in \mathbb{H} \mid(\Im \tau-\eta) / 2 \leqslant \Im z \leqslant(\Im \tau+\eta) / 2\} \subset \mathbb{H}$ is a horizontal strip which projects to an annular neighbourhood $A$ of $h_{\infty} \subset \mathbb{P}$. Let $S \subset \mathbb{P}$ be the surface $\mathbb{P}$ with the projection of the open horocyclic neighbourhood $\Im z>(\Im \tau+\eta) / 2$ of $\infty$ deleted. Define $h_{\infty}^{\prime}, S^{\prime}$ and $A^{\prime}$ in a similar way. We are going to glue $S$ to $S^{\prime}$ by matching $A$ to $A^{\prime}$ in such a way that $h_{\infty}$ is identified to $h_{\infty}^{\prime}$ with orientation reversed; see Figure 10. The resulting homotopy class of the loop $h_{\infty}$ on the glued up surface (the quotient of the disjoint union of the surfaces $S_{i}$ by the attaching maps across the $A_{i}$ ) will be in the homotopy class of $\sigma$. To keep track of the marking on $\Sigma$, we will do the gluing on the level of the $\mathbb{Z}$-covers of $S, S^{\prime}$ corresponding to $h_{\infty}, h_{\infty}^{\prime}$, that is, we will actually glue the strips $H_{\infty}$ and $H_{\infty}^{\prime}$.

As shown in Figure 10, the deleted punctured disks are on opposite sides of $h_{\infty}$ in $S$ and $h_{\infty}^{\prime}$ in $S^{\prime}$. Thus we first need to reverse the direction in one of the two strips $H_{\infty}, H_{\infty}^{\prime}$. Set

$$
J=\left(\begin{array}{cc}
-i & 0 \\
0 & i
\end{array}\right), \quad T_{\tau}=\left(\begin{array}{ll}
1 & \tau \\
0 & 1
\end{array}\right)
$$

We reverse the direction in $H_{\infty}$ by applying the map $J(z)=-z$ to $\mathbb{H}$. We then glue $H_{\infty}$ to $H_{\infty}^{\prime}$ by identifying $z \in H_{\infty}$ to $z^{\prime}=T_{\tau} J(z) \in H_{\infty}^{\prime}$. Since both $J$ and $T_{\tau}$ commute with the holonomies $z \mapsto z+2$ and $z^{\prime} \mapsto z^{\prime}+2$ of the curves $h_{\infty}, h_{\infty}^{\prime}$, this identification descends to a well defined identification of $A$ with $A^{\prime}$, in which the "outer" boundary $\zeta(\Im z)=(\Im \tau+\eta) / 2$ of $A$ is identified to the "inner" boundary $\zeta^{\prime}\left(\Im z^{\prime}\right)=(\Im \tau-\eta) / 2$ of $A^{\prime}$. In particular, $h_{\infty}$ is glued to $h_{\infty}^{\prime}$ reversing orientation.

Now we treat the general case in which $P$ and $P^{\prime}$ meet along punctures with arbitrary labels $\epsilon, \epsilon^{\prime} \in\{0,1, \infty\}$. As above, let $\Delta_{0} \subset \mathbb{H}$ be the ideal "white" triangle with vertices $0,1, \infty$. Notice that there is a unique orientation preserving symmetry $\Omega_{\alpha}$ of $\Delta_{0}$ which sends the vertex $\alpha \in\{0,1, \infty\}$ to $\infty$ :

$$
\Omega_{0}=\left(\begin{array}{cc}
1 & -1 \\
1 & 0
\end{array}\right), \quad \Omega_{1}=\left(\begin{array}{ll}
0 & -1 \\
1 & -1
\end{array}\right), \quad \Omega_{\infty}=\mathrm{Id}=\left(\begin{array}{ll}
1 & 0 \\
0 & 1
\end{array}\right) .
$$

Let $h_{\epsilon}$ be the loop on $\mathbb{P}$ which lifts to the horocycle $\Omega_{\epsilon}^{-1}(\{z \in \mathbb{H} \mid \Im z=\Im \tau / 2\})$ on $\mathbb{H}$, so that $h_{\epsilon}$ is a loop round $\partial_{\epsilon}(P)$. Also let $H_{\epsilon}$ be the region in $\mathbb{H}$ defined by $\Omega_{\epsilon}^{-1}(\{z \in \mathbb{H} \mid(\Im \tau-\eta) / 2 \leqslant \Im z \leqslant(\Im \tau+\eta) / 2\})$. Define $h_{\epsilon^{\prime}}^{\prime}$ and $H_{\epsilon^{\prime}}^{\prime}$ in a similar way. 
To do the gluing, first move $\epsilon, \epsilon^{\prime}$ to $\infty$ using the maps $\Omega_{\epsilon}, \Omega_{\epsilon^{\prime}}$ and then proceed as before. Thus the gluing identifies $z \in H_{\epsilon}$ to $z^{\prime} \in H_{\epsilon}^{\prime}$ by the formula

$$
\Omega_{\epsilon^{\prime}}\left(z^{\prime}\right)=T_{\tau} \circ J\left(\Omega_{\epsilon}(z)\right)
$$

(see Figure 10).

Finally, we carry out the above construction for each pants curve $\sigma_{i} \in \mathcal{P C}$. To do this, we need to ensure that the annuli corresponding to the three different punctures of a given $\mathbb{P}_{i}$ are disjoint. Note that the condition $\Im \tau_{i}>2$, for all $i=1, \ldots, \xi$, ensures that the three curves $h_{0}, h_{1}$ and $h_{\infty}$ associated to the three punctures of $P_{i}$ are disjoint in $\mathbb{P}$. Under this condition, we can clearly choose $\eta>0$ so that their annular neighbourhoods are disjoint, as required.

In what follows, we shall usually write $h, H$ for $h_{\epsilon}, H_{\epsilon}$ provided the subscript is clear from the context.

Remark 4.1 Note that in the above construction of $\Sigma_{\tau}$, we glued a curve exiting from the white triangles $\Delta_{0}(P)$ to one entering the white triangle $\Delta_{0}\left(P^{\prime}\right)$. Suppose that we wanted instead to glue the two black triangles $\Delta_{1}(P)$ and $\Delta_{1}\left(P^{\prime}\right)$. This can be achieved by replacing the parameter $\tau$ with $\tau-2$. However, following our recipe, it is not possible to glue a curve exiting a white triangle to a curve entering a black one, because the black triangle is to the right of both the outgoing and incoming lines while the white triangle is to the left.

4.1.1 Independence of the direction of the travel The recipe for gluing two pants apparently depends on the direction of travel across their common boundary. The following lemma shows that, in fact, the gluing in either direction is implemented by the same recipe and uses the same parameter $\tau$.

Lemma 4.2 Let pants $P$ and $P^{\prime}$ be glued across a common boundary $\sigma$, and suppose the gluing used when travelling from $P$ to $P^{\prime}$ is implemented by (3) with the parameter $\tau$. Then the gluing when travelling in the opposite direction from $P^{\prime}$ to $P$ is also implemented by (3) with the same parameter $\tau$.

Proof Using the maps $\Omega_{\epsilon}$ if necessary, we may, without loss of generality, suppose we are gluing the boundary $\partial_{\infty} P$ to $\partial_{\infty} P^{\prime}$. (Note that $\Omega_{\infty}=$ Id.) By definition, to do this we identify the horocyclic strip $H \subset \mathbb{H}$ to the strip $H^{\prime} \subset \mathbb{H}^{\prime}$ using the map $T_{\tau} \circ J$.

Fix a point $X \in h$. The gluing sends $X$ to $T_{\tau} J(X) \in h^{\prime}$. The gluing in the other direction from $P^{\prime}$ to $P$ reverses orientation of the strips to be glued and is done using a translation $T_{\tau^{\prime}}$ say. To give the same gluing we must have $T_{\tau^{\prime}} J T_{\tau} J(X)=X$. This gives $\tau^{\prime}-(-X+\tau)=X$ which reduces to $\tau=\tau^{\prime}$ as claimed. 


\subsection{Marking and Dehn twists}

Write $\underline{\tau}=\left(\tau_{1}, \ldots, \tau_{\xi}\right) \in \mathbb{C}^{\xi}$, and denote by $\Sigma(\underline{\tau})$ the surface obtained by the gluing procedure described above with parameter $\tau_{i}$ along curve $\sigma_{i}$. To complete the description of the projective structure, we have to specify a marking on $\Sigma(\underline{\tau})$. To do this we have to specify a base structure on a fixed surface $\Sigma_{0}$, together with a homeomorphism $\Phi_{\underline{\tau}}: \Sigma_{0} \rightarrow \Sigma(\underline{\tau})$.

We first fix the base structure on $\Sigma_{0}$, together with a marking given by a family of dual curves $D_{i}$ to the pants curves $\sigma_{i}$. Let $\mu_{\epsilon} \subset \mathbb{H}$ be the unique oriented geodesic from $\epsilon+1$ to $\epsilon+2$, where $\epsilon$ is in the cyclically ordered set $\{0,1, \infty\}$; see Figure 9 . The lines $\mu_{\epsilon}$ project to the seams of $\mathbb{P}$. We call $\mu_{0}$ (from 1 to $\infty$ ) and $\mu_{1}$ (from $\infty$ to 0 ) respectively the incoming and the outgoing strands (coming into and going out from the puncture) at $\infty$, and refer to their images under the maps $\Omega_{\epsilon}$ in a similar way. For $\tau \in \mathbb{C}$, let $X_{\infty}(\tau)=1+\Im \tau / 2$ be the point at which the incoming line $\mu_{0}$

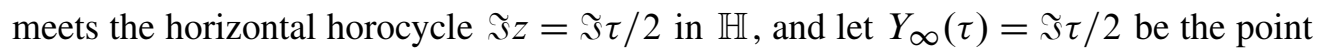
the outgoing line $\mu_{1}$ meets the same horocycle. Also define $X_{\epsilon}(\tau)=\Omega_{\epsilon}\left(X_{\infty}\right)$ and $Y_{\epsilon}(\tau)=\Omega_{\epsilon}\left(Y_{\infty}\right)$. Now pick a pants curve $\sigma$ and, as usual, let $P, P^{\prime} \in \mathcal{P}$ be its adjacent pants in $\Sigma$, to be glued across boundaries $\partial_{\epsilon} P$ and $\partial_{e^{\prime}} P^{\prime}$. Let $X_{\epsilon}(P), X_{\epsilon}\left(P^{\prime}\right)$ be the points corresponding to $X_{\epsilon}(\tau), X_{\epsilon}(\tau)$ under the identifications $\Phi, \Phi^{\prime}$ of $P, P^{\prime}$ with $\mathbb{P}$, and similarly for $Y_{\epsilon}(P), Y_{\epsilon}\left(P^{\prime}\right)$. The base structure $\Sigma_{0}$ will be one in which the identification (3) matches the point $X_{\epsilon}(P, \tau)$ on the incoming line across $\partial_{\epsilon} P$ to the point $Y_{\epsilon^{\prime}}^{\prime}\left(P^{\prime}\right)$ on the outgoing line to $\partial_{\epsilon^{\prime}} P^{\prime}$. Referring to the gluing Equation (3), we see that this condition is fulfilled precisely when $\Re \tau=1$.

We define the structure on $\Sigma_{0}$ by specifying $\Re \tau_{i}=1$ for $i=1, \ldots, \xi$. The imaginary part of $\tau_{i}$ is unimportant; for definiteness we can fix $\Im \tau_{i}=4$. Now note that the reflection $z \mapsto-\bar{z}$ of $\mathbb{H}$ induces an orientation reversing isometry of $\mathbb{P}$ which fixes its seams; with the gluing matching seams as above this extends, in an obvious way, to an orientation reversing involution of $\Sigma_{0}$. Following (a) of Section 3.1.1, this specification is equivalent to a specification of a marking on $\Sigma_{0}$.

Finally, we define a marking on the surface $\Sigma(\underline{\tau})$. After applying a suitable stretching to each pants to adjust the lengths of the boundary curves, we can map $\Sigma_{0} \rightarrow \Sigma(\underline{\tau})$ using a map which is the Fenchel-Nielsen twist $\mathrm{FN}_{\Re \tau_{i}}$ on an annulus around $\sigma_{i} \in \mathcal{P C}$, $i=1, \ldots, \xi$ and the identity elsewhere; see Section 3.1.1. This gives a well defined homotopy class of homeomorphisms $\Psi_{\underline{\tau}}: \Sigma_{0} \rightarrow \Sigma(\underline{\tau})$.

With this description, it is easy to see that $\Re \tau_{i}$ corresponds to twisting about $\sigma_{i}$; in particular, $\tau_{i} \mapsto \tau_{i}+2$ is a full right Dehn twist about $\sigma_{i}$. The imaginary part $\Im \tau_{i}$ corresponds to vertical translation and has the effect of scaling the lengths of the $\sigma_{i}$. 


\subsection{Projective structure and holonomy}

The above construction gives a way to define the developing map from the universal cover $\tilde{\Sigma}$ of $\Sigma$ to $\mathbb{C}$. To do this we have to describe the developing image of any path $\gamma$ on $\Sigma$. The path goes through a sequence of pants $P_{i_{1}}, P_{i_{2}}, \ldots, P_{i_{n}}$ such that each adjacent pair $P_{i_{j}}, P_{i_{j+1}}$ is glued along an annular neighbourhood $A\left(\sigma_{i_{j}}\right)$ of the pants curve $\sigma_{i_{j}}$ which forms the common boundary of $P_{i_{j}}$ and $P_{i_{j+1}}$. Since all the maps involved in this gluing are in $\operatorname{PSL}(2, \mathbb{C})$, it is clear that if $\gamma$ is a closed loop, then the holonomy of $\gamma$ is in $\operatorname{PSL}(2, \mathbb{C})$. Thus we get a representation $\pi_{1}(\Sigma) \rightarrow \operatorname{PSL}(2, \mathbb{C})$ which can be checked to be independent of $\gamma$ up to homotopy in the usual way.

Now we can justify our claim that our construction gives a projective structure on $\Sigma$. Recall that a complex projective structure on $\Sigma$ means an open covering of $\Sigma$ by simply connected sets $U_{i}$, such that $U_{i} \cap U_{j}$ is connected and simply connected, together with homeomorphisms $\Phi_{i}: U_{i} \rightarrow V_{i} \subset \widehat{\mathbb{C}}$, such that the overlap maps $\Phi_{i} \circ \Phi_{j}^{-1}: \Phi_{j}\left(U_{i} \cap U_{j}\right) \rightarrow \Phi_{i}\left(U_{i} \cap U_{j}\right)$ are in $\operatorname{PSL}(2, \mathbb{C})$.

Given the developing map $\Psi: \widetilde{\Sigma} \rightarrow \widehat{\mathbb{C}}$ from the universal covering space $\widetilde{\Sigma}$ of $\Sigma$ into the Riemann sphere $\widehat{\mathbb{C}}$, we can clearly cover $\Sigma$ by open sets $U_{i}$ such that each component $W$ of the lift of $U_{i}$ to $\tilde{\Sigma}$ is homeomorphic to $U_{i}$ and such that the restriction $\left.\Psi\right|_{W}$ is a homeomorphism to an open set $V \subset \widehat{\mathbb{C}}$. For each $U_{i}$, pick one such component. Since any two lifts differ by a covering map, and since $U_{i} \cap U_{j} \neq \varnothing$ implies there are lifts which intersect, the overlap maps will always be in the covering group which by our construction is contained in $\operatorname{PSL}(2, \mathbb{C})$.

In terms of the projective structure, the holonomy representation $\rho: \pi_{1}(\Sigma) \rightarrow \operatorname{PSL}(2, \mathbb{C})$ is described as follows. A path $\gamma$ in $\Sigma$ passes through an ordered chain of charts $U_{0}, \ldots, U_{n}$ such that $U_{i} \cap U_{i+1} \neq \varnothing$ for every $i=0, \ldots, n-1$. This gives us the overlap maps $R_{i}=\Phi_{i} \circ \Phi_{i+1}^{-1}$ for $i=0, \ldots, n-1$. The sets $V_{i}$ and $R_{i}\left(V_{i+1}\right)$ overlap in $\widehat{\mathbb{C}}$ and hence the developing image of $\tilde{\gamma}$ in $\widehat{\mathbb{C}}$ passes through in order the sets $V_{0}, R_{0}\left(V_{1}\right), R_{0} R_{1}\left(V_{2}\right), \ldots, R_{0} \cdots R_{n-1}\left(V_{n}\right)$. If $\gamma$ is closed, we have $U_{n}=U_{0}$ so that $V_{0}=V_{n}$. Then, by definition, the holonomy of the homotopy class $[\gamma]$ is $\rho([\gamma])=R_{0} \cdots R_{n-1} \in \operatorname{PSL}(2, \mathbb{C})$.

Notice that our construction effectively takes the charts to be the maps $P_{i} \rightarrow \mathbb{H}$ which identify $P_{i}$ with the standard fundamental domain $\Delta \subset \mathbb{H}$ via the map $\Phi_{i}: P_{i} \rightarrow \mathbb{P}$. Strictly speaking, we should divide each $P_{i}$ into two simply connected sets by cutting along its seams, so that each chart maps to one or other of the standard ideal triangles $\Delta_{0}$ or $\Delta_{1}$. The details of how this works will be discussed in Section 5.

As a consequence of the construction we note the following fact which underlies the connection with the Maskit embedding (Section 4.5), and which (together with the definition of the twist in the case $q_{i}=0$ ) proves the final statement of Theorem A. 
Lemma 4.3 Suppose that $\gamma \in \pi_{1}(\Sigma)$ is a loop homotopic to a pants curve $\sigma_{j}$. Then $\rho(\gamma)$ is parabolic and $\operatorname{Tr} \rho(\gamma)= \pm 2$.

\subsection{Relation to Kra's plumbing construction}

Kra in [5] uses essentially the above construction to manufacture surfaces by gluing triply punctured spheres across punctures, a procedure which he calls plumbing. Plumbing is based on so called "horocyclic coordinates" in punctured disk neighbourhoods of the punctures which have to be glued.

Given a puncture $\epsilon$ on a triply punctured sphere $\mathbb{P}$, let $\zeta: \mathbb{H} \rightarrow \mathbb{P}$ be the natural projection, normalised so that $\epsilon$ lifts to $\infty \in \mathbb{H}$, and so that the holonomy of the loop round $\epsilon$ is, as above, $\eta \mapsto \eta+2$. Let $\mathbb{D}_{*}$ denote the punctured unit disc $\{z \in \mathbb{C}$ : $0<z<1\}$. The function $f: \mathbb{H} \rightarrow \mathbb{D}_{*}$ given by $f(\eta)=e^{i \pi \eta}$ is well defined in a neighbourhood $N$ of $\infty$ and is a homeomorphism from an open neighbourhood of $\epsilon$ in $\mathbb{P}$ to an open neighbourhood of the puncture in $\mathbb{D}_{*}$. Choosing another puncture $\epsilon^{\prime}$ of $\mathbb{P}$, we can further normalise so that $\epsilon^{\prime}$ lifts to 0 . Hence $f$ maps the part of the geodesic from $\epsilon^{\prime}$ to $\epsilon$ contained in $N$, to the interval $(0, r)$ for suitable $r>0$. These normalisations (which depend only on the choices of $\epsilon$ and $\epsilon^{\prime}$ ), uniquely determine $f$. Kra calls the natural parameter $z=f(\eta)$ in $\mathbb{D}_{*}$, the horocyclic coordinate of the puncture $\epsilon$ relative to $\epsilon^{\prime}$.

Now suppose that $\widehat{z}$ and $\widehat{z}^{\prime}$ are horocyclic coordinates for distinct punctures in distinct copies $\mathbb{P}_{\widehat{z}}$ and $\mathbb{P}_{\widehat{z}^{\prime}}$ of $\mathbb{P}$. Denote the associated punctured discs by $\mathbb{D}_{*}(\widehat{z})$ and $\mathbb{D}_{*}\left(\widehat{z}^{\prime}\right)$. To plumb across the two punctures, first delete punctured disks $\{0<\widehat{z}<r\}$ and $\left\{0<\widehat{z}^{\prime}<r^{\prime}\right\}$ from $\mathbb{D}_{*}(\widehat{z})$ and $\mathbb{D}_{*}\left(\widehat{z}^{\prime}\right)$ respectively. Then glue the remaining surfaces along the annuli

$$
A(\widehat{z})=\left\{\widehat{z} \in \mathbb{D}_{*}: r<\widehat{z}<s\right\} \quad \text { and } \quad A\left(\hat{z}^{\prime}\right)=\left\{\hat{z}^{\prime} \in \mathbb{D}_{*}: r^{\prime}<\hat{z}^{\prime}<s^{\prime}\right\}
$$

by the formula $\widehat{z} \widehat{z}^{\prime}=t_{K}$. (To avoid confusion we have written $t_{K}$ for Kra's parameter $t \in \mathbb{C}$.) It is easy to see that this is essentially identical to our construction; the difference is simply that we implement the gluing in $\mathbb{H}$ and $\mathbb{H}^{\prime}$ without first mapping to $\mathbb{D}_{*}(\widehat{z})$ and $\mathbb{D}_{*}\left(\widehat{z}^{\prime}\right)$. Our method has the advantage of having a slightly simpler formula and also of respecting the twisting around the puncture, which is lost under the map $f$.

The precise relation between our coordinates $z, z^{\prime} \in \mathbb{H}$ in Section 4.1 and the horocyclic coordinates $\widehat{z}, \hat{z}^{\prime}$ is

$$
z=f^{-1}(\widehat{z})=-\frac{i}{\pi} \log \widehat{z}, \quad z^{\prime}=f^{-1}\left(\widehat{z}^{\prime}\right)=-\frac{i}{\pi} \log \widehat{z}^{\prime} .
$$


The relation

$$
\widehat{z} \widehat{z}^{\prime}=t_{K}
$$

translates to

$$
\log t_{K}=\log \widehat{z}^{\prime}+\log \widehat{z}
$$

which, modulo $2 \pi i \mathbb{Z}$, is exactly our relation

$$
-z+\tau=z^{\prime}
$$

Hence we deduce that

$$
\tau=-\frac{i}{\pi} \log t_{K}
$$

\subsection{Relation to the Maskit embedding of $\Sigma$}

As usual let $\mathcal{P C}=\left\{\sigma_{1}, \ldots, \sigma_{\xi}\right\}$ be a pants decomposition of $\Sigma$. We have constructed a family of projective structures on $\Sigma$, to each of which is associated a natural holonomy representation $\rho_{\underline{\tau}}: \pi_{1}(\Sigma) \rightarrow \operatorname{PSL}(2, \mathbb{C})$. We want to prove that our construction, for suitable values of the parameters, gives exactly the Maskit embedding of $\Sigma$. For the definition of this embedding we follow the second author [13]; see also Maskit [9]. Let $\mathcal{R}(\Sigma)$ be the representation variety of $\pi_{1}(\Sigma)$, that is, the set of representations $\rho: \pi_{1}(\Sigma) \rightarrow \operatorname{PSL}(2, \mathbb{C})$ modulo conjugation in $\operatorname{PSL}(2, \mathbb{C})$. Let $\mathcal{M} \subset \mathcal{R}$ be the subset of representations for which

(i) the group $G=\rho\left(\pi_{1}(\Sigma)\right)$ is discrete (Kleinian) and $\rho$ is an isomorphism;

(ii) the images of $\sigma_{i}, i=1, \ldots, \xi$, are parabolic;

(iii) all components of the regular set $\Omega(G)$ are simply connected and there is exactly one invariant component $\Omega^{+}(G)$;

(iv) the quotient $\Omega(G) / G$ has $k+1$ components (where $k=2 g-2+n$ if $\Sigma=$ $\left.\Sigma_{(g, n)}\right), \Omega^{+}(G) / G$ is homeomorphic to $\Sigma$ and the other components are triply punctured spheres.

In this situation, see for example Marden [8, Section 3.8], the corresponding 3-manifold $M_{\rho}=\mathbb{H}^{3} / G$ is topologically $\Sigma \times(0,1)$. Moreover $G$ is a geometrically finite cusp group on the boundary (in the algebraic topology) of the set of quasifuchsian representations of $\pi_{1}(\Sigma)$. The "top" component $\Omega^{+} / G$ of the conformal boundary may be identified to $\Sigma \times\{1\}$ and is homeomorphic to $\Sigma$. On the "bottom" component $\Omega^{-} / G$, identified to $\Sigma \times\{0\}$, the pants curves $\sigma_{1}, \ldots, \sigma_{\xi}$ have been pinched, making $\Omega^{-} / G$ a union of $k$ triply punctured spheres glued across punctures corresponding to the curves $\sigma_{i}$. The conformal structure on $\Omega^{+} / G$, together with the pinched curves $\sigma_{1}, \ldots, \sigma_{\xi}$, are the end invariants of $M_{\rho}$ in the sense of Minsky's ending 
lamination theorem. Since a triply punctured sphere is rigid, the conformal structure on $\Omega^{-} / G$ is fixed and independent of $\rho$, while the structure on $\Omega^{+} / G$ varies. It follows from standard Ahlfors-Bers theory, using the measurable Riemann mapping theorem (see again Marden [8, Section 3.8]), that there is a unique group corresponding to each possible conformal structure on $\Omega^{+} / G$. Formally, the Maskit embedding of the Teichmüller space of $\Sigma$ is the map $\mathcal{T}(\Sigma) \rightarrow \mathcal{R}$ which sends a point $X \in \mathcal{T}(\Sigma)$ to the unique group $G \in \mathcal{M}$ for which $\Omega^{+} / G$ has the marked conformal structure $X$.

Proposition 4.4 Suppose that $\underline{\tau} \in \mathbb{C}^{\xi}$ is such that the associated developing map $\operatorname{Dev}_{\tau}: \widetilde{\Sigma} \rightarrow \widehat{\mathbb{C}}$ is an embedding. Then the holonomy representation $\rho_{\underline{\tau}}$ is a group isomorphism and $G=\rho_{\tau}\left(\pi_{1}(\Sigma)\right) \in \mathcal{M}$.

Proof Since the developing map Dev: $\widetilde{\Sigma} \rightarrow \widehat{\mathbb{C}}$ is an embedding, $G=\rho_{\underline{\tau}}\left(\pi_{1}(\Sigma)\right)$ is Kleinian. By construction (see Lemma 4.3), the holonomy of each of the curves $\sigma_{1}, \ldots, \sigma_{\xi}$ is parabolic. This proves (i) and (ii).

The image of Dev is a simply connected $G$-invariant component $\Omega^{+}=\operatorname{Dev}(\tilde{\Sigma})$ of the regular set $\Omega(G)$ of $G$. Since $\Omega^{+}$is $G$-invariant, its boundary $\partial \Omega^{+}$is the limit set $\Lambda(G)$.

Let $P \in \mathcal{P}$, and let $\widetilde{P}$ be a lift of $P$ to the universal cover $\widetilde{\Sigma}$. The boundary curves $\sigma_{i_{1}}, \sigma_{i_{2}}, \sigma_{i_{3}}$ of $P$ lift, in particular, to three curves in $\partial \widetilde{P}$ corresponding to elements $\gamma_{i_{1}}, \gamma_{i_{2}}, \gamma_{i_{3}} \in \pi_{1}(\Sigma)$ such that $\gamma_{i_{1}} \gamma_{i_{2}} \gamma_{i_{3}}=$ id and such that $\rho\left(\gamma_{i_{j}}\right)$ is parabolic for $j=1,2,3$. These generate a subgroup $\Gamma(\widetilde{P})$ of $S L(2, \mathbb{R})$ conjugate to $\Gamma$; see Section 4.1. Thus the limit set $\Lambda(\widetilde{P})$ of $\Gamma(\widetilde{P})$ is a round circle $C(\widetilde{P})$.

Without loss of generality, fix the normalisation of $G$ such that $\infty \in \Omega^{+}(G)$. Since $\Omega^{+}(G)$ is connected, it must be contained in the component of $\widehat{\mathbb{C}} \backslash \Lambda(\widetilde{P})$ which contains $\infty$. Since $\Lambda(G)=\partial \Omega^{+}(G)$, we deduce that $\Lambda(G)$ is also contained in the closure of the same component, and hence that the open disk $D(\widetilde{P})$ bounded by $C(\widetilde{P})$ and not containing $\infty$, contains no limit points. (In the terminology of [4], $\Gamma(\widetilde{P})$ is peripheral with peripheral disk $D(\widetilde{P})$.) It follows that $D(\widetilde{P})$ is precisely invariant under $\Gamma(\widetilde{P})$ and hence that $D(\widetilde{P}) / G=D(\widetilde{P}) / \Gamma(\widetilde{P})$ is a triply punctured sphere.

Thus $\Omega(G) / G$ contains the surface $\Sigma(G)=\Omega^{+}(G) / G$ and the union of $k$ triply punctured spheres $D(\widetilde{P}) / \Gamma(\widetilde{P})$, one for each pair of pants in $\mathcal{P}$. Thus the total hyperbolic area of $\Omega(G) / G$ is at least $4 \pi k$. Now Bers' area inequality [1] (see also eg Matsuzaki and Taniguchi [10, Theorem 4.6]) states that

$$
\operatorname{Area}(\Omega(G) / G) \leq 4 \pi(T-1)
$$


where $T$ is the minimal number of generators of $G$, in our case $2 g+b-1$. Since $k=2 g+b-2$ we have

$$
4 \pi(2 g+b-2) \leq \operatorname{Area}(\Omega(G) / G) \leq 4 \pi(T-1)=4 \pi(2 g+b-2) .
$$

We deduce that $\Omega(G)$ is the disjoint union of $\Omega^{+}(G)$ and the disks $D(\widetilde{P}), P \in \mathcal{P}$. This completes the proof of (iii) and (iv).

This gives an alternative viewpoint on our main result: we are finding a formula for the leading terms in $\tau_{i}$ of the trace polynomials of simple curves on $\Sigma$ under the Maskit embedding of $\mathcal{T}(\Sigma)$. This was the context in which the result was presented in [4; 13]; see also Section 5.3.1.

\section{Calculation of paths}

In this section we discuss how to compute the holonomy of some simple paths. We first specify a particular path joining one hexagon to the next, then we study paths contained in one pair of pants, and finally we compute the holonomy representations of some paths in the one holed torus and in the four times punctured sphere.

The gluing construction in Section 4 effectively takes the charts to be the maps which identify $P_{i}$ with the standard fundamental domain $\Delta \subset \mathbb{H}$. Precisely, as explained above, for each $P=P_{i} \in \mathcal{P}$, we have a fixed homeomorphism $\Phi_{i}: P \rightarrow \mathbb{P}$ and hence a $\operatorname{map} \zeta^{-1} \circ \Phi_{i}: P \rightarrow \mathbb{H}$, where $\zeta$ is the projection of $\mathbb{H}$ to $\mathbb{P}$. Let $\Delta_{0}(P)=\Phi_{i}^{-1} \circ \zeta\left(\Delta_{0}\right)$ be the white hexagon in $P$. Also let $b(P)=\Phi_{i}^{-1} \circ \zeta\left(b_{0}\right)$ where $b_{0}=(1+i \sqrt{3}) / 2 \in \Delta_{0}$ is the barycentre of the white triangle. This will serve as a base point in $\Delta_{0}(P)$.

Suppose that $\gamma \in \mathcal{S}$. Although not logically necessary, we can greatly simplify our description by arranging $\gamma$ in standard Penner position, so that it always passes from one pants to the next through the white hexagons $\Delta_{0}\left(P_{i}\right)$. Suppose, as in Section 4.3, that $\gamma$ passes through a sequence of pants $P_{i_{1}}, \ldots, P_{i_{n}}$. We may as well assume that $\gamma$ starts at the base point $b\left(P_{i_{1}}\right)$ of $P_{i_{1}}$. Given our identification $\Phi_{i_{1}}$ of $P_{i_{1}}$ with $\mathbb{P}$, there is a unique lift $\tilde{b}\left(P_{i_{1}}\right) \in \Delta_{0}$ and hence there is a unique lift $\tilde{\gamma}$ of $\gamma \cap P_{i_{1}}$ to $\mathbb{H}$ starting at $\tilde{b}\left(P_{i_{1}}\right)$. This path exits $\Delta_{0}$ either across one of its three sides, or across that part of a horocycle which surrounds one of the three cusps $0,1, \infty$ contained in $\Delta_{0}$. In the first case, the holonomy is given by the usual action of the group $\Gamma$ on $\mathbb{H}$, where $\Gamma$ is the triply punctured sphere group as in Section 4.1. (This will be explained in detail in Section 5.2.) In the second case, we have a precise description of the gluing across the boundary annuli, giving a unique way to continue $\tilde{\gamma}$ into a lift of $P_{i_{2}}$. In this case we continue in a new chart in which the lift of $P_{i_{2}}$ is identified with $\Delta \subset \mathbb{H}$, as before. 
The following result applies to an arbitrary connected loop on $\Sigma$.

Proposition 5.1 Let $\gamma \in \pi_{1}(\Sigma)$ and suppose that $\sum_{i} i\left(\gamma, \sigma_{i}\right)=q$. Then the trace $\operatorname{Tr} \rho([\gamma])$ is a polynomial in $\tau_{1}, \ldots, \tau_{\xi}$ of maximal total degree $q$ and of maximal degree $q_{k}(\gamma)=i\left(\gamma, \sigma_{k}\right)$ in the parameter $\tau_{k}$.

Proof Suppose the boundary $\partial_{\epsilon} P$ of one pair of pants $P$ is glued to the boundary $\partial_{\epsilon^{\prime}} P^{\prime}$ of another pair $P^{\prime}$ along a pants curve $\sigma$. The map $\Phi_{P^{\prime}} \Phi_{P}^{-1}: \mathbb{H}_{P} \rightarrow \mathbb{H}_{P^{\prime}}$ which glues the horocycle labelled $\epsilon$ in $\Delta_{0}(P)$ to the horocycle labelled $\epsilon^{\prime}$ in $\Delta_{0}\left(P^{\prime}\right)$ is $\Omega_{\epsilon^{\prime}}^{-1} T_{\tau} J \Omega_{\epsilon}$, where as usual the maps $\Omega_{\epsilon}$ and $\Omega_{\epsilon^{\prime}}$ are the standard maps taking $\epsilon, \epsilon^{\prime}$ to $\infty$. Thus with the notation of Section 4.3, the overlap map $R=\Phi_{P} \Phi_{P^{\prime}}^{-1}$ is

$$
\Omega_{\epsilon}^{-1} J^{-1} T_{\tau}^{-1} \Omega_{\epsilon^{\prime}}
$$

Any curve $\gamma \in \pi_{1}(\Sigma)$ which intersects the pants curves $\sigma_{i}$ in total $q$ times passes through a sequence of pants $P_{i_{1}}, \ldots, P_{i_{q}}=P_{i_{1}}$ and can therefore be written as a product $\prod_{j=1}^{q} \kappa_{j} \lambda_{j}$ where $\kappa_{j} \in \pi_{1}\left(P_{i_{j}} ; b_{i_{j}}\right)$ is a path in $P_{i_{j}}$ with both its endpoints in the base point $b_{i_{j}}=b\left(P_{i_{j}}\right)$ and $\lambda_{j}=\lambda\left(P_{i_{j}}, P_{i_{j+1}}\right)$ is a path from $b_{i_{j}}$ to $b_{i_{j+1}}$ across the boundary $\sigma_{i_{j}}$ between $P_{i_{j}}$ and $P_{i_{j+1}}$.

Let $\rho: \pi_{1}\left(P_{i_{j}} ; b_{i_{j}}\right) \rightarrow \Gamma$ be the map induced by the identification of $P_{i_{j}}$ with $\Delta \subset \mathbb{H}$, where $\Gamma$ is the triply punctured sphere group as in Section 4.1. It follows that the holonomy of $\gamma$ is a product

$$
\rho([\gamma])=\prod_{j=1}^{q} \rho\left(\kappa_{j}\right) \Omega_{\epsilon_{j}}^{-1} J^{-1} T_{\tau_{i_{j}}}^{-1} \Omega_{\epsilon_{j+1}}^{\prime}
$$

from which the result follows.

It is clear from this formula that $\operatorname{Tr} \rho([\gamma])$ is an invariant of the free homotopy class of $\gamma$, because changing the base point of the path $\gamma$ changes the above product by conjugation.

We can also define the holonomy of paths with distinct endpoints in one pair of pants $P$; see Section 5.2.

\subsection{Paths between adjacent pants}

Suppose that pants $P$ and $P^{\prime}$ are glued along $\sigma \in \mathcal{P C}$. If $\partial_{\infty} P$ is glued to $\partial_{\infty} P^{\prime}$, then there is an obvious path $\lambda\left(P, P^{\prime} ; \infty, \infty ; 0\right)$ on $\Sigma_{0}$ from $b(P)$ to $b\left(P^{\prime}\right)$, namely the projection to $\Sigma_{0}$ of the union of the path $z=b(P)+i t, t \in[0, \Im \tau / 2+\epsilon]$ in $\Delta_{0}(P)$ with the path $z^{\prime}=b\left(P^{\prime}\right)+i t, t \in[\Im \tau / 2+\epsilon, 0]$ in $\Delta_{0}\left(P^{\prime}\right)$. More generally, if $\partial_{\epsilon} P$ is 
glued to $\partial_{\epsilon^{\prime}} P^{\prime}$, we define $\lambda\left(P, P^{\prime} ; \epsilon, \epsilon^{\prime} ; 0\right)$ to be the path obtained by first applying the maps $\Omega_{\epsilon}, \Omega_{\epsilon^{\prime}}$ to the segments of $\lambda\left(P, P^{\prime} ; \infty, \infty ; 0\right)$ in $P, P^{\prime}$ respectively. For a general surface $\Sigma(\underline{\tau})$ we define $\lambda\left(P, P^{\prime} ; \epsilon, \epsilon^{\prime} ; \underline{\tau}\right)=\Phi_{\underline{\tau}}\left(\lambda\left(P, P^{\prime} ; \epsilon, \epsilon^{\prime} ; 0\right)\right)$. Note that $\lambda\left(P, P^{\prime}, \sigma ; \underline{\tau}\right)$ is entirely contained in the white triangles in $P$ and $P^{\prime}$. Unless needed for clarity, we refer to all these paths as $\lambda\left(P, P^{\prime}\right)$.

Referring to the gluing Equation (3) we see that the holonomy of $\lambda\left(P, P^{\prime}\right)$ is given by

$$
\rho\left(\lambda\left(P, P^{\prime} ; \epsilon, \epsilon^{\prime} ; \underline{\tau}\right)\right)=\Omega_{\epsilon}^{-1} J^{-1} T_{\tau}^{-1} \Omega_{\epsilon^{\prime}} .
$$

As already noted in Lemma 4.2, the gluing parameters $\underline{\tau}$ are independent of the direction of travel (from $P$ to $P^{\prime}$ or vice versa). From (6) we have

$$
\rho\left(\lambda\left(P^{\prime}, P ; \epsilon^{\prime}, \epsilon ; \underline{\tau}\right)\right)=\Omega_{\epsilon^{\prime}}^{-1} J^{-1} T_{\tau}^{-1} \Omega_{\epsilon}
$$

so that

$$
\rho\left(\lambda\left(P^{\prime}, P ; \epsilon^{\prime}, \epsilon ; \underline{\tau}\right)^{-1}\right)=\Omega_{\epsilon}^{-1} T_{\tau} J \Omega_{\epsilon^{\prime}} .
$$

Using the identities $J^{-1}=-J, T_{\tau}^{-1}=T_{-\tau}$ and $T_{\tau} J=J T_{-\tau}$ this gives

$$
\rho\left(\lambda\left(P^{\prime}, P ; \epsilon^{\prime}, \epsilon ; \underline{\tau}\right)^{-1}\right)=-\rho\left(\lambda\left(P^{\prime}, P ; \epsilon^{\prime}, \epsilon ; \underline{\tau}\right)\right)^{-1},
$$

as one would expect. That fact will be particularly important for our proof in Section 6 .

\subsection{Paths in a pair of pants}

We now calculate the holonomy of the three boundary loops in one pair of pants $P$. As usual, we identify $P$ with $\mathbb{P}$ so that the components of its boundary are labelled $0,1, \infty$ in some order, and the base point is the barycentre $b_{0}=(1+\sqrt{3} i) / 2$ of $\Delta_{0}$. Orient each of the three boundary curves $\partial_{\epsilon}(\mathbb{P})$, where $\epsilon \in\{0,1, \infty\}$, consistently with the three lines $\mu_{\epsilon}$ where, as above, $\mu_{\epsilon} \subset \mathbb{H}$ is the unique oriented geodesic from $\epsilon+1$ to $\epsilon+2$, where $\epsilon$ is in the cyclically ordered set $\{0,1, \infty\}$; see Figure 9 . We denote by $\eta_{\epsilon} \in \pi_{1}\left(P ; b_{0}\right)$ the loop based at $b_{0}$ and freely homotopic to the oriented loop $\partial_{\epsilon}(\mathbb{P})$. To calculate the holonomy of $\eta_{\epsilon}$, we begin by noting the holonomies of the three homotopically distinct paths $\gamma_{\epsilon}$, with $\epsilon \in\{0,1, \infty\}$, joining $b_{0}$ to $b_{0}^{*}=(-1+\sqrt{3} i) / 2$, the barycentre of $\Delta_{1}$; see Figure 11 .

Each path $\gamma_{\epsilon}$ is determined by the geodesic $\mu_{\epsilon}$ which it crosses. Thus $\gamma_{0}$ connects $b_{0}$ and $b_{0}^{*}$ crossing $\mu_{0}$, and so on. The holonomies of these three paths are

$$
\rho\left(\gamma_{0}\right)=\left(\begin{array}{ll}
1 & 2 \\
0 & 1
\end{array}\right), \quad \rho\left(\gamma_{1}\right)=\mathrm{Id}=\left(\begin{array}{ll}
1 & 0 \\
0 & 1
\end{array}\right), \quad \rho\left(\gamma_{\infty}\right)=\left(\begin{array}{ll}
1 & 0 \\
2 & 1
\end{array}\right),
$$

as is clear from Figure 11. 


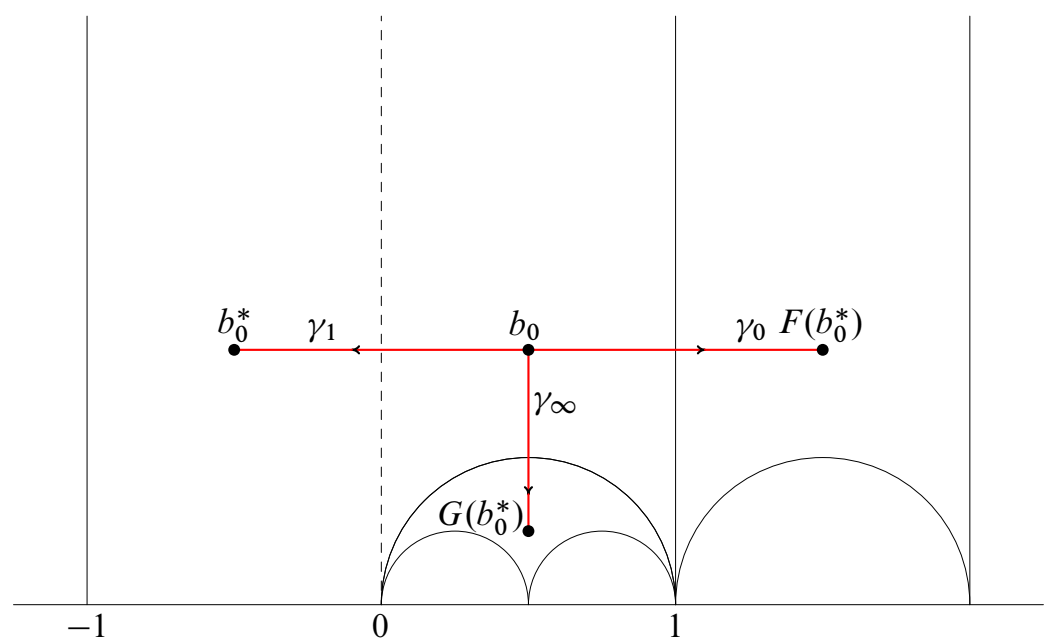

Figure 11: Paths between $b_{0}$ and $b_{0}^{*}$ where $F=\rho\left(\gamma_{0}\right)$ and $G=\rho\left(\gamma_{\infty}\right)$

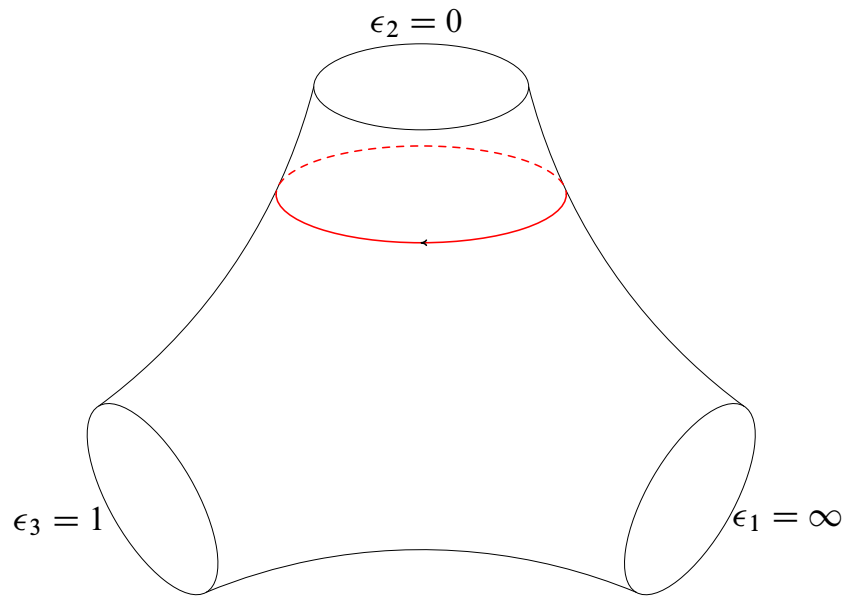

Figure 12: The loop $\eta_{0}$ homotopic to $\partial_{0} P$

To calculate the holonomy of the loop $\eta_{0}$ around the boundary $\partial_{0} P$, we have to go from $b_{0}$ to $b_{0}^{*}$ crossing $\mu_{\infty}$ and then go from $b_{0}^{*}$ to $b_{0}$ crossing $\mu_{1}$. Hence, as illustrated in Figures 11 and 12, we have to go along the path $\gamma_{\infty}$ and then along the path $\gamma_{1}^{-1}$. Thus we find

$$
\rho\left(\eta_{0}\right)=\rho\left(\gamma_{\infty} \gamma_{1}^{-1}\right)=\left(\begin{array}{ll}
1 & 0 \\
2 & 1
\end{array}\right) .
$$

Similarly to calculate the holonomy of $\eta_{1}$, we have to go from $b_{0}$ and $b_{0}^{*}$ crossing $\mu_{0}$ and then return from $b_{0}^{*}$ to $b_{0}$ crossing $\mu_{\infty}$. This means going along $\gamma_{0}$ and then $\gamma_{\infty}^{-1}$. 
Thus the holonomy is

$$
\rho\left(\eta_{1}\right)=\rho\left(\gamma_{0} \gamma_{\infty}^{-1}\right)=\left(\begin{array}{ll}
1 & 2 \\
0 & 1
\end{array}\right)\left(\begin{array}{cc}
1 & 0 \\
-2 & 1
\end{array}\right)=\left(\begin{array}{ll}
-3 & 2 \\
-2 & 1
\end{array}\right) .
$$

Finally, to calculate the holonomy of $\eta_{\infty}$, we have to go from $b_{0}$ to $b_{0}^{*}$ crossing $\mu_{1}$ and return from $b_{0}^{*}$ to $b_{0}$ crossing $\mu_{0}$. Hence we have to go along the path $\gamma_{1}$ and then along the path $\gamma_{0}^{-1}$, so the holonomy is

$$
\rho\left(\eta_{\infty}\right)=\rho\left(\gamma_{1} \gamma_{0}^{-1}\right)=\left(\begin{array}{cc}
1 & -2 \\
0 & 1
\end{array}\right)
$$

As a check, we verify that

$$
\rho\left(\eta_{0}\right) \rho\left(\eta_{\infty}\right) \rho\left(\eta_{1}\right)=\left(\begin{array}{ll}
1 & 0 \\
2 & 1
\end{array}\right)\left(\begin{array}{cc}
1 & -2 \\
0 & 1
\end{array}\right)\left(\begin{array}{ll}
-3 & 2 \\
-2 & 1
\end{array}\right)=\mathrm{Id}
$$

in accordance with the relation $\eta_{0} \eta_{\infty} \eta_{1}=\mathrm{id}$ in $\pi_{1}\left(\mathbb{P} ; b_{0}\right)$.

\subsection{Examples}

To conclude this section, we look at the special cases of the once punctured torus and the four times punctured sphere.

5.3.1 The once punctured torus The once punctured torus $\Sigma_{1,1}$ is decomposed into one pair of pants by cutting along a single pants curve $\sigma$. To determine the projective structure on $\Sigma_{1,1}$ following our construction, we take a pair of pants $P$ and glue the boundaries $\partial_{\infty} P$ and $\partial_{0} P$, so that the remaining boundary $\partial_{1} P$ becomes the puncture on $\Sigma_{1,1}$; see Figure 13 . To find $\rho: \pi_{1}\left(\Sigma_{1,1}\right) \rightarrow \operatorname{PSL}(2, \mathbb{C})$, it is sufficient to compute the holonomy of $\sigma$ and of its dual curve $D_{\sigma}$.

To do the gluing, take two copies of $P$ and, following the notation in Section 4, label the copy on the left in the figure $P$, and that on the right, $P^{\prime}$. We identify $P$ with the standard triply punctured sphere $\mathbb{P}$ by the homeomorphism $\Phi: P \rightarrow \mathbb{P}$ so that the universal covers $\widetilde{P}, \widetilde{P}^{\prime}$ are identified with copies $\mathbb{H}, \mathbb{H}^{\prime}$ of the upper half plane $\mathbb{H}$ with coordinates $z, z^{\prime}$ respectively. The cusps to be glued are labelled $\epsilon=\infty$ and $\epsilon^{\prime}=0$. We first apply the standard symmetries $\Omega_{\epsilon}, \Omega_{\epsilon^{\prime}}$ which carry $\epsilon=\infty$ and $\epsilon^{\prime}=0$ to $\infty$. Referring to (2), we see that $\Omega_{\infty}(z)=z$ and $\Omega_{0}\left(z^{\prime}\right)=1-1 / z^{\prime}$.

According to the choices made in Section 3.1.1, the dual curve $D_{\sigma}$ to $\sigma$ is the curve $\lambda\left(P, P^{\prime}, \sigma ; \tau\right)$ joining $b(P) \in P$ to $b\left(P^{\prime}\right) \in P^{\prime}$. By (5) in Section 5 and by the 


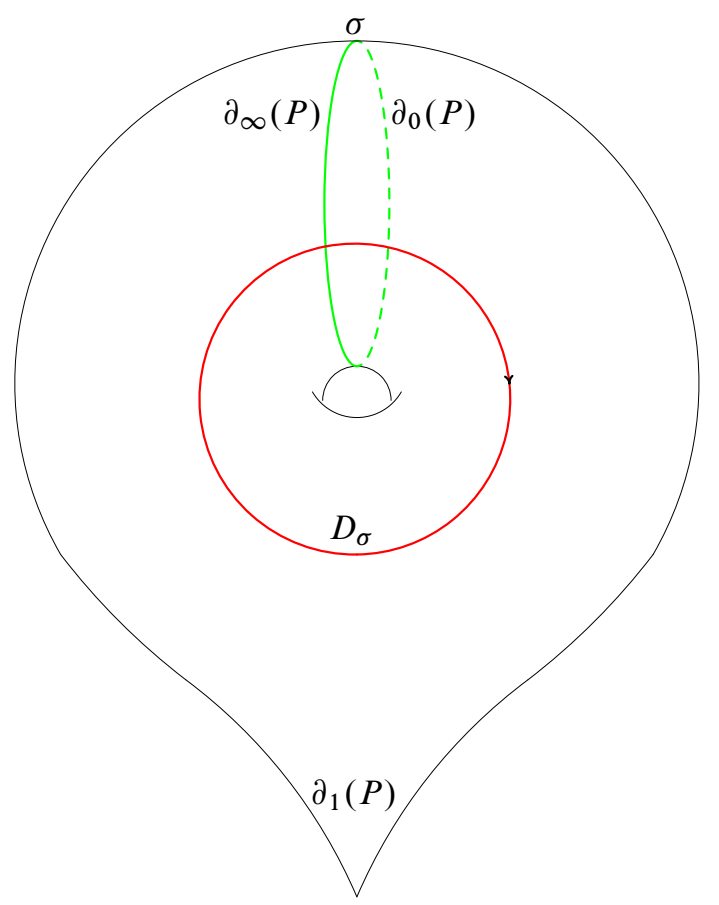

Figure 13: Plumbing for the once punctured torus

formulae (2) for the standard symmetries, we have

$$
\begin{aligned}
\rho\left(D_{\sigma}\right) & =\Omega_{\infty}^{-1} J^{-1} T_{\tau}^{-1} \Omega_{0} \\
& =\left(\begin{array}{cc}
i & 0 \\
0 & -i
\end{array}\right)\left(\begin{array}{cc}
1 & -\tau \\
0 & 1
\end{array}\right)\left(\begin{array}{cc}
1 & -1 \\
1 & 0
\end{array}\right)=-i\left(\begin{array}{cc}
\tau-1 & 1 \\
1 & 0
\end{array}\right) .
\end{aligned}
$$

Since clearly

$$
\rho(\sigma)=\left(\begin{array}{ll}
1 & 2 \\
0 & 1
\end{array}\right)
$$

this is enough to specify the representation $\rho: \pi_{1}(\Sigma) \rightarrow \operatorname{PSL}(2, \mathbb{C})$.

The original motivation for studying the representations in this paper came from studying the Maskit embedding of the once punctured torus; see Keen and Series [4] and Section 4.5. The Maskit embedding for $\Sigma_{1,1}$ is described in [4] as the representation $\rho^{\prime}: \pi_{1}\left(\Sigma_{1,1}\right) \rightarrow \operatorname{PSL}(2, \mathbb{C})$ given by

$$
\rho^{\prime}(\sigma)=\left(\begin{array}{ll}
1 & 2 \\
0 & 1
\end{array}\right) \quad \text { and } \quad \rho^{\prime}\left(D_{\sigma}\right)=-i\left(\begin{array}{cc}
\mu & 1 \\
1 & 0
\end{array}\right) \text {. }
$$

This agrees with the above formula setting $\mu=\tau-1$. 
5.3.2 The four holed sphere $\Sigma_{0,4}$ We decompose $\Sigma_{0,4}$ into two pairs of pants $P$ and $P^{\prime}$ by cutting along the curve $\sigma$, and label the boundary components as shown in Figure 14, so that the boundaries to be glued are both labelled $\infty$. In the figure, $P$ is the upper of the two pants and $P^{\prime}$ the lower. We shall calculate the holonomy of the dual $D_{\sigma}$ to $\sigma$ in two different ways, first in the standard Penner position and secondly in the symmetrical DT-position. As it is to be expected, the two calculations give the same result.

The loop $D_{\sigma}$ in Penner standard position If we put the loop $D_{\sigma}$ in Penner standard position, as illustrated in Figure 5, and as described in Section 3.2, we see that it the concatenation of the paths

(1) $\lambda\left(P, P^{\prime} ; \infty, \infty ; \tau\right)$ from $b_{0}(P)$ to $b_{0}\left(P^{\prime}\right)$;

(2) $\eta_{0}\left(P^{\prime}\right)$;

(3) $\eta_{\infty}\left(P^{\prime}\right)$;

(4) $\lambda\left(P^{\prime}, P ; \infty, \infty ; \tau\right)$ from $b_{0}\left(P^{\prime}\right)$ to $b_{0}(P)$;

(5) $\eta_{0}^{-1}(P)$.

Thus using the calculations in Sections 5.1 and 5.2, we have

$$
\begin{aligned}
\rho\left(D_{\sigma}\right) & =\rho\left(\lambda\left(P, P^{\prime} ; \infty, \infty ; \tau\right) \cdot \eta_{0}\left(P^{\prime}\right) \cdot \eta_{\infty}\left(P^{\prime}\right) \cdot \lambda\left(P^{\prime}, P ; \infty, \infty ; \tau\right) \cdot \eta_{0}^{-1}(P)\right) \\
& =\left(\begin{array}{cc}
i & -i \tau \\
0 & -i
\end{array}\right) \cdot\left(\begin{array}{ll}
1 & 0 \\
2 & 1
\end{array}\right) \cdot\left(\begin{array}{cc}
1 & -2 \\
0 & 1
\end{array}\right) \cdot\left(\begin{array}{cc}
-i & i \tau \\
0 & i
\end{array}\right) \cdot\left(\begin{array}{cc}
1 & 0 \\
-2 & 1
\end{array}\right) \\
& =\left(\begin{array}{cc}
-4 \tau^{2}+6 \tau-3 & 2 \tau^{2}-4 \tau+2 \\
-4 \tau+4 & 2 \tau-3
\end{array}\right)
\end{aligned}
$$

giving

$$
\operatorname{Tr} \rho\left(D_{\sigma}\right)=-4 \tau^{2}+8 \tau-6
$$

Now $q\left(D_{\sigma}\right)=2$ and $p\left(D_{\sigma}\right)=0$ (see Section 3.1), and the number $h$ of scc-arcs in $D_{\sigma}$ is 2 . Thus Theorem A predicts that

$$
\operatorname{Tr} \rho\left(D_{\sigma}\right)= \pm i^{2} 2^{2}(\tau+(0-2) / 2)^{2}+R
$$

where $R$ represents terms of degree at most 0 in $\tau$, in accordance with the computation above. 


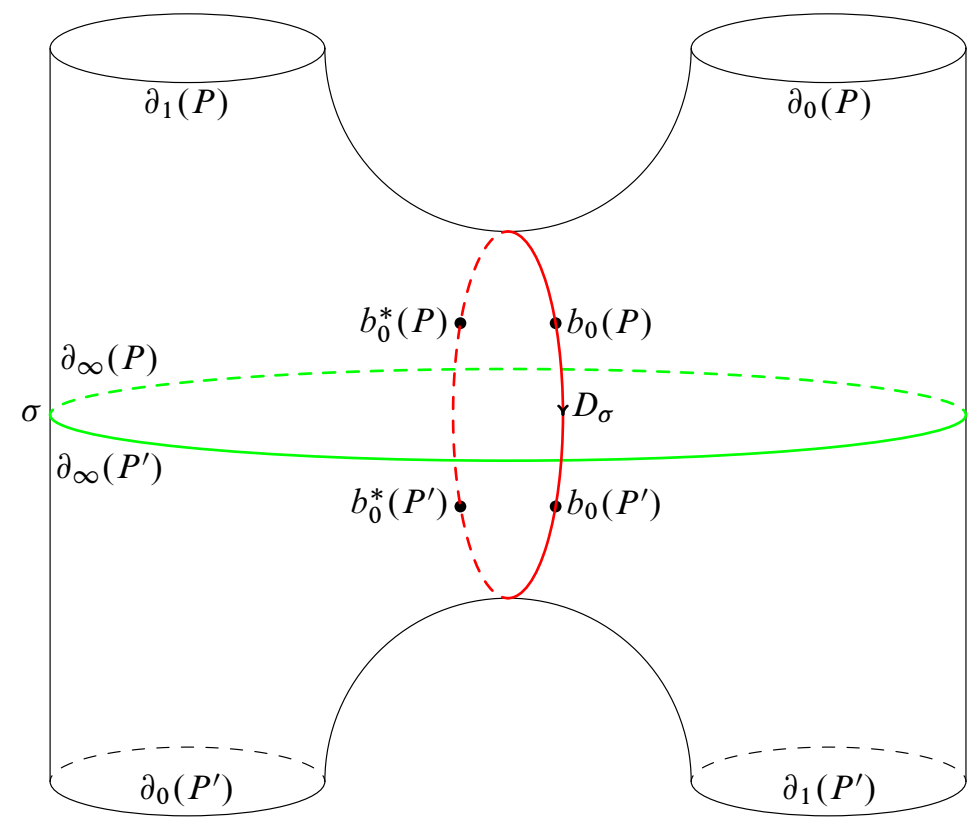

Figure 14: The loop $D_{\sigma}$ in its symmetrical DT-position in the four holed sphere

The loop $D_{\sigma}$ in symmetrical DT-position As usual we take as base points the barycenters $b_{0}(P)$ and $b_{0}^{*}(P)$ of the "white" and the "black" hexagons respectively in $P$ and the same base points $b_{0}\left(P^{\prime}\right)$ and $b_{0}^{*}\left(P^{\prime}\right)$ in $P^{\prime}$. Also denote $\lambda^{*}\left(P, P^{\prime} ; \infty, \infty ; \tau\right)$ the path $R\left(\lambda\left(P, P^{\prime} ; \infty, \infty ; \tau\right)\right)$ from $b_{0}^{*}(P)$ to $b_{0}^{*}\left(P^{\prime}\right)$ through the black hexagons, where $R$ is the orientation reversing symmetry of $\Sigma(\tau)$ as in Section 3.1.

From Figure 14, we see that $D_{\sigma}$ is the concatenation of the paths

(1) $\lambda\left(P, P^{\prime} ; \infty, \infty ; \tau\right)$ from $b_{0}(P)$ to $b_{0}\left(P^{\prime}\right)$;

(2) $\gamma_{\infty}\left(P^{\prime}\right)$ in $P^{\prime}$ from $b_{0}\left(P^{\prime}\right)$ to $b_{0}^{*}\left(P^{\prime}\right)$;

(3) $\lambda^{*}\left(P^{\prime}, P ; \infty, \infty ; \tau\right)$ from $b_{0}^{*}\left(P^{\prime}\right)$ to $b_{0}^{*}(P)$;

(4) $\gamma_{\infty}^{-1}(P)$ in $P$ from $b_{0}^{*}(P)$ to $b_{0}(P)$.

Thus

$$
\rho\left(D_{\sigma}\right)=\rho\left(\lambda\left(P, P^{\prime}, \sigma ; \tau\right)\right) \cdot \rho\left(\gamma_{\infty}(P)\right) \cdot \rho\left(\lambda^{*}\left(P^{\prime}, P, \sigma ; \tau\right)\right) \cdot \rho\left(\gamma_{\infty}^{-1}(P)\right) .
$$

Following Remark 4.1 we have

$$
\lambda^{*}\left(P^{\prime}, P ; \infty, \infty ; \tau\right)=\lambda\left(P^{\prime}, P ; \infty, \infty ; \tau-2\right)=\lambda\left(P, P^{\prime} ; \infty, \infty ; \tau-2\right)^{-1}
$$


so that, from Section 5.1, we have

$$
\rho\left(\lambda^{*}\left(P^{\prime}, P ; \infty, \infty ; \tau\right)\right)=\left(\begin{array}{cc}
-i & i(\tau-2) \\
0 & i
\end{array}\right) .
$$

Thus referring also to Section 5.2 we see

$$
\begin{aligned}
\rho\left(D_{\sigma}\right) & =\left(\begin{array}{cc}
i & -i \tau \\
0 & -i
\end{array}\right) \cdot\left(\begin{array}{ll}
1 & 0 \\
2 & 1
\end{array}\right) \cdot\left(\begin{array}{cc}
-i & i(\tau-2) \\
0 & i
\end{array}\right) \cdot\left(\begin{array}{cc}
1 & 0 \\
-2 & 1
\end{array}\right) \\
& =\left(\begin{array}{cc}
-4 \tau^{2}+6 \tau-3 & 2 \tau^{2}-4 \tau+2 \\
-4 \tau+4 & 2 \tau-3
\end{array}\right) .
\end{aligned}
$$

Hence $\operatorname{Tr}\left(\rho\left(D_{\sigma}\right)\right)=-4 \tau^{2}+8 \tau-6$ as before.

\section{Proof of Theorem A}

In this final section we prove Theorem A. Our method is to show that the product of matrices forming the holonomy always takes a special form and then give an inductive proof.

First consider the holonomy representation of a typical path. Let $\gamma \in \mathcal{S}_{0}$ be a simple loop on $\Sigma$. We suppose $\gamma$ is in Penner standard position, so that it always cuts $\sigma_{i_{j}}$ in the arc $w_{i_{j}}$. Starting from the basepoint in some pants $P$, it crosses, in order, pants curves $\sigma_{i_{j}}, j=1, \ldots, q(\gamma)$. If the boundaries glued across $\sigma_{i_{j}}$ are $\partial_{\epsilon} P, \partial_{\epsilon^{\prime}} P^{\prime}$, then, by Equation (4), the contribution to the holonomy product $\rho(\gamma)$ is

$$
\Omega_{\epsilon}^{-1} J^{-1} T_{\tau_{i}}^{-1} \Omega_{\epsilon^{\prime}}^{\prime}
$$

where $\tau_{i_{j}}=\tau_{i}$ whenever $\sigma_{i_{j}}=\sigma_{i} \in \mathcal{P C}$.

A single positive twist around $\partial_{\epsilon} P$ immediately before this boundary component contributes $\rho\left(\eta_{\epsilon}^{-1}\right)=\Omega_{\epsilon}^{-1} \rho\left(\eta_{\infty}^{-1}\right) \Omega_{\epsilon}$ (because $\eta_{\epsilon}$ twists in the negative direction round $\partial_{\epsilon} P$; see Figure 12), while a single positive twist around $\partial_{\epsilon^{\prime}} P^{\prime}$ after the crossing contributes $\rho\left(\eta_{\epsilon}\right)=\Omega_{\epsilon^{\prime}}^{-1} \rho\left(\eta_{\infty}\right) \Omega_{\epsilon^{\prime}}$. Thus if, in general, $\gamma$ twists $\alpha_{j}$ times around $\partial_{\epsilon} P=\sigma_{i_{j}}$ immediately before the crossing and $\beta_{j}$ times after, the total contribution to the holonomy is

$$
\Omega_{\epsilon}^{-1} \rho\left(\eta_{\infty}\right)^{-\alpha_{j}} J^{-1} T_{\tau_{i}}^{-1} \rho\left(\eta_{\infty}\right)^{\beta_{j}} \Omega_{\epsilon^{\prime}}^{\prime}
$$

where $\sigma_{i_{j}}=\sigma_{i} \in \mathcal{P C}$.

From Sections 4.1 and 5.2 we have

$$
J^{-1} T_{\tau}^{-1}=\left(\begin{array}{cc}
i & -i \tau \\
0 & -i
\end{array}\right) \text { and } \rho\left(\eta_{\infty}\right)=\left(\begin{array}{cc}
1 & -2 \\
0 & 1
\end{array}\right)
$$


For variables $X, Y$, write

$$
A_{X}=\left(\begin{array}{cc}
1 & X \\
0 & -1
\end{array}\right) \text { and } B_{Y}=\left(\begin{array}{ll}
1 & Y \\
0 & 1
\end{array}\right)
$$

We calculate

$$
\rho\left(\eta_{\infty}\right)^{-\alpha_{j}} J^{-1} T_{\tau_{i}}^{-1} \rho\left(\eta_{\infty}\right)^{\beta_{j}}=i A_{X_{j}}
$$

with $X_{j}=-\left(\tau_{i}+2 \alpha_{j}+2 \beta_{j}\right)$, from which we note in particular that, as expected, which side of the crossing the twists occur makes no difference to the final product.

Proposition 6.1 $\quad$ (i) Suppose that $\gamma$ contains no scc-arcs. Then $\rho(\gamma)$ is of the form $\pm i^{q} \prod_{i=1}^{q} A_{X_{j}} \Omega_{i_{j}}$, where $\Omega_{i_{j}}=\Omega_{0}$ or $\Omega_{1}$ for all $j$. If the term $A_{X_{j}}$ corresponds to the crossing of a pants curve $\sigma_{i_{j}}=\sigma_{i}$, with $\alpha_{j}$ twists before the crossing and $\beta_{j}$ after, then $X_{j}=-\left(\tau_{i}+2 \alpha_{j}+2 \beta_{j}\right)$.

(ii) If $\gamma$ contains scc-arcs, then $\rho(\gamma)$ takes the same form as above, with an extra term

$$
A_{X_{j}} \Omega_{1} B_{ \pm 2} \Omega_{0} A_{X_{j}}
$$

inserted for each scc-arc which crosses $\sigma_{i_{j}}$ twice in succession.

(iii) In all cases, the total P-twist of $\gamma$ about $\sigma_{i} \in \mathcal{P C}$ is $\hat{p}_{i}(\gamma)=\sum_{\sigma_{i_{j}}=\sigma_{i}}\left(\alpha_{j}+\beta_{j}\right)$.

Proof As computed above we have

$$
\rho\left(\eta_{\infty}\right)^{-\alpha} J^{-1} T_{\tau}^{-1} \rho\left(\eta_{\infty}\right)^{\beta}=i\left(\begin{array}{cc}
1 & -(\tau+2 \alpha+2 \beta) \\
0 & -1
\end{array}\right)=i A_{-(\tau+2 \alpha+2 \beta)} .
$$

So the holonomy $\rho(\gamma)$ is a concatenation of $q$ terms of the form $i \Omega_{\epsilon}^{-1} A_{-(\tau+2 \alpha+2 \beta)} \Omega_{\epsilon^{\prime}}$, one for each crossing of a pants curve $\sigma_{i_{j}}$. If $\gamma$ contains no scc-arcs, then it enters and leaves each pants $P$ across distinct boundary components, say $\partial_{\epsilon_{1}} P$ and $\partial_{\epsilon_{2}} P$ respectively. Then the corresponding adjacent terms in the concatenated product are then

$$
\cdots \Omega_{\epsilon_{1}} \Omega_{\epsilon_{2}}^{-1} \ldots
$$

where $\epsilon_{1} \neq \epsilon_{2}$, from which (i) easily follows.

We also note that regardless of how the twists are organised before or after the crossings, the sum $\sum_{j=1}^{q_{i}(\gamma)}\left(\alpha_{j}+\beta_{j}\right)$ of coefficients in terms corresponding to crossings of the pants curve $\sigma_{i}$ is equal to $\hat{p}_{i}(\gamma)$, the $i$-th P-twisting number of the curve $\gamma$ with respect to the pants decomposition $\mathcal{P C}$. This proves (iii).

Now suppose that $\gamma$ contains some scc-arcs. Suppose that $\gamma$ cuts a curve $\sigma_{i_{j}}$ twice in succession entering and leaving a pants $P$ across the boundary $\partial_{\infty} P$. Since $\gamma$ is in $\mathrm{P}$-form, after crossing $\partial_{\infty} P$ it goes once around $\partial_{0} P$ in either the positive or 
negative direction and then returns to $\partial_{\infty} P$; see Figure 5. Since $\gamma$ is simple, the twisting around $\sigma_{i_{j}}$ is the same on the inward and the outward journeys. The term in the holonomy is therefore

$$
A_{X_{j}} \rho\left(\eta_{0}\right)^{ \pm 1} A_{X_{j}}=A_{X_{j}} \Omega_{0}^{-1} \rho\left(\eta_{\infty}\right)^{\mp 1} \Omega_{0} A_{X_{j}}=A_{X_{j}} \Omega_{0}^{-1} B_{ \pm 2} \Omega_{0} A_{X_{j}}
$$

as claimed.

If more generally $\gamma$ enters and leaves $P$ across $\partial_{\epsilon} P$, then this entire expression is multiplied on the left by $\Omega_{\epsilon}^{-1}$ and on the right by $\Omega_{\epsilon}$. By the same discussion as in (i), this leaves the form of the holonomy product unchanged. The contribution to the twist about $\sigma_{i_{j}}$ is calculated as before.

We are now ready for our inductive proof of Theorem A. Suppose first that $\gamma \in \mathcal{S}_{0}$ contains no scc-arcs. If

$$
\rho(\gamma)= \pm i^{q} \prod_{i=1}^{q} A_{X_{j}} \Omega_{i_{j}}
$$

define $X_{j}^{*}=X_{j}+h\left(\Omega_{i_{j}}\right)+k\left(\Omega_{i_{j-1}}\right)$ where $\Omega_{i_{0}}:=\Omega_{i_{q}}$ and

$$
h\left(\Omega_{i_{j}}\right)=\left\{\begin{array}{ll}
1 & \text { if } i_{j}=0, \\
0 & \text { otherwise, }
\end{array} \quad \text { and } \quad k\left(\Omega_{i_{j}}\right)= \begin{cases}0 & \text { if } i_{j}=0, \\
1 & \text { otherwise }\end{cases}\right.
$$

Thus

$$
\begin{aligned}
& \Omega_{0} A_{X} \Omega_{0} \rightarrow X^{*}=X+1 \\
& \Omega_{0} A_{X} \Omega_{1} \rightarrow X^{*}=X \\
& \Omega_{1} A_{X} \Omega_{0} \rightarrow X^{*}=X+2 \\
& \Omega_{1} A_{X} \Omega_{1} \rightarrow X^{*}=X+1 .
\end{aligned}
$$

Remark 6.2 Replacing $\rho(\gamma)$ by $\rho(\gamma)^{-1}$ leaves the occurrences of the above blocks unchanged. The entire matrix product is multiplied by $(-1)^{q}$. This is because $A_{X}^{-1}=$ $-A_{X}$ and, for example,

$$
\left(\Omega_{0} A_{X} \Omega_{1}\right)^{-1}=\Omega_{1}^{-1} A_{X}^{-1} \Omega_{0}^{-1}=-\Omega_{0} A_{X} \Omega_{1} .
$$

Now given the path of some $\gamma \in \mathcal{S}_{0}$, consider a crossing for which $\sigma_{i_{j}}=\sigma_{i}$. Let $\Omega_{i_{j-1}} A_{X_{j}} \Omega_{i_{j}}$ be the corresponding terms in $\rho(\gamma)$, (where $\Omega_{i_{j-1}}$ is associated to the crossing of the previous pants curve $\sigma_{i_{j-1}}$ ). Let $p_{j}, \hat{p}_{j}$ be the respective contributions from this $j$-th crossing to the DT- and P-twist coordinates of $\gamma$, so that the total twists $p_{i}, \hat{p}_{i}$ about $\sigma_{i}$ are obtained by summing over all crossings for which $\sigma_{i_{j}}=\sigma_{i}$ : $p_{i}=\sum_{\sigma_{i_{j}}=\sigma_{i}} p_{j}$ and likewise $\hat{p}_{i}=\sum_{\sigma_{i_{j}}=\sigma_{i}} \hat{p}_{j}$. 
For any variable $a_{j} \in \mathbb{R}$ which depends on the $j$-th crossing, define $a_{j}^{*}$ according to the same rule as $X^{*}$ above, in other words $a_{j}^{*}=a_{j}+h\left(\Omega_{i_{j}}\right)+k\left(\Omega_{i_{j-1}}\right)$. We have:

Lemma 6.3 Suppose that $\gamma$ contains no scc-arcs and as usual let $p_{j}, \hat{p}_{j}$ be the contributions to the DT- and P-twists of $\gamma$ corresponding to the $j$-th crossing of a pants curve $\sigma_{i} \in \mathcal{P C}$. Then $\left(-2 \hat{p}_{j}\right)^{*}=-p_{j}+1$.

Proof This is verified using Theorem 3.5, together with the fact that $\gamma$ is assumed to be in $\mathrm{P}-$ standard form.

Consider a crossing for which $\sigma_{i_{j}}=\sigma_{i}$ with corresponding term $\Omega_{i_{j-1}} A_{X_{j}} \Omega_{i_{j}}$ in $\rho(\gamma)$. Suppose for example that the relevant term in the holonomy is $\Omega_{0} A_{X_{j}} \Omega_{0}$, so that by definition, $a_{j}^{*}=a_{j}+1$ for any variable $a_{j}$. Without loss of generality, we may suppose that $\sigma_{i_{j}}$ is the gluing of $\partial_{\infty} P$ to $\partial_{\infty} P^{\prime}$ as shown in Figure 6. The first $\Omega_{0}$ means that there is an arc from $D$ to $E$, and the second $\Omega_{0}$ means there is an arc from $E$ to $A$. The formula of Theorem 3.5 therefore gives a contribution $2 \hat{p}_{j}=p_{j}+0+1-1=p_{j}$. Thus $\left(-2 \hat{p}_{j}\right)^{*}:=-2 \hat{p}_{j}+1=-p_{j}+1$ as claimed.

Similarly, consider the sequence $\Omega_{1} A_{X_{j}} \Omega_{0}$. In this case, $\left(-2 \hat{p}_{j}\right)^{*}=-2 \hat{p}_{j}+2$. From Theorem 3.5 we find $2 \hat{p}_{j}=p_{j}+1+1-1=p_{j}+1$, so $\left(-2 \hat{p}_{j}\right)^{*}=-2 \hat{p}_{j}+2=-p_{j}+1$.

The other two possible sequences are similar.

In the case of no scc-arcs, Theorem A is an immediate corollary of this lemma and the following proposition:

Proposition 6.4 Suppose that $\gamma$ contains no scc-arcs, then

$$
\operatorname{Tr}(\rho(\gamma))= \pm i^{q} \operatorname{Tr}\left(\prod_{j=1}^{q} A_{X_{j}} \Omega_{i_{j}}\right)= \pm i^{q}\left(\prod_{j=1}^{q} X_{j}^{*}\right)+R
$$

where $R$ denotes terms of degree at most $q-2$ in the $X_{j}$.

Proof of Theorem A (No scc-arcs case) By Proposition 6.1, if $\sigma_{i_{j}}=\sigma_{i}$ then $X_{j}=$ $-\left(\tau_{i}+2 \alpha_{j}+2 \beta_{j}\right)$. There are $q_{i}(\gamma)$ such terms $X_{j}$. Thus the top order term of $\operatorname{Tr} \rho(\gamma)$ is $\tau_{1}^{q_{1}} \cdots \tau_{\xi}^{q_{\xi}}$, with coefficient $\pm i^{q}$, in accordance with the result of Theorem A. 
Now the contribution to the $\mathrm{P}$-twist corresponding to the $j$-th crossing is $\hat{p}_{j}=\alpha_{j}+\beta_{j}$. Thus

$$
\begin{aligned}
\prod_{j=1}^{q} X_{j}^{*} & =\prod_{j=1}^{q}\left(-\left(\tau_{i}+2 \alpha_{j}+2 \beta_{j}\right)\right)^{*} \\
& =\prod_{j=1}^{q}\left(-\left(\tau_{i}+2 \hat{p}_{j}\right)\right)^{*} \\
& =\prod_{j=1}^{q}\left(-\tau_{i}+\left(-2 \hat{p}_{j}\right)^{*}\right) \\
& =\prod_{j=1}^{q}\left(-\tau_{i}-p_{j}+1\right) \\
& =(-1)^{q} \prod_{j=1}^{q}\left(\tau_{i}+p_{j}-1\right)
\end{aligned}
$$

where we used Lemma 6.3 to evaluate $\left(-2 p_{j}\right)^{*}$. So the coefficient of $\tau_{1}^{q_{1}} \cdots \tau_{i}^{q_{i}-1} \cdots \tau_{\xi}^{q_{\xi}}$ is

$$
\pm i^{q} \sum_{\sigma_{i_{j}}=\sigma_{i}}\left(p_{j}-1\right)= \pm i^{q}\left(p_{i}-q_{i}\right)
$$

which is exactly the coefficient in Theorem A.

Proof of Proposition 6.4 We prove this by induction on the length $q$ of the product $\prod_{j=1}^{q} A_{X_{j}} \Omega_{i_{j}}$. If $q=1$, with respect to the cyclic ordering we see either the block $\Omega_{0} A_{X} \Omega_{0}$ or $\Omega_{1} A_{X} \Omega_{1}$, so that $X^{*}=X+1$. In both cases we check directly that $\operatorname{Tr} A_{X} \Omega_{0}=\operatorname{Tr} A_{X} \Omega_{1}=X+1$.

The case $q=2$ corresponds to a product $A_{X_{1}} \Omega_{\epsilon} A_{X_{2}} \Omega_{\epsilon^{\prime}}$. Hence there are four possibilities corresponding to $\epsilon= \pm 1$ and $\epsilon^{\prime}= \pm 1$. These cases can be checked either by multiplying out or by using the trace identity

$$
\operatorname{Tr}(A B)=\operatorname{Tr}(A) \operatorname{Tr}(B)-\operatorname{Tr}\left(A B^{-1}\right) .
$$

For example, if $\epsilon=\epsilon^{\prime}=0$, then

$$
\begin{aligned}
\operatorname{Tr} A_{X_{1}} \Omega_{0} A_{X_{2}} \Omega_{0} & =\operatorname{Tr}\left(A_{X_{1}} \Omega_{0}\right) \operatorname{Tr}\left(A_{X_{2}} \Omega_{0}\right)+\operatorname{Tr} A_{X_{1}} A_{X_{2}} \\
& =\left(X_{1}+1\right)\left(X_{2}+1\right)+2=X_{1}^{*} X_{2}^{*}+2,
\end{aligned}
$$

where we used the relation $A_{X}^{-1}=-A_{X}$. 
If $\epsilon=0, \epsilon^{\prime}=1$ then

$$
\operatorname{Tr}\left(A_{X_{1}} \Omega_{0} A_{X_{2}} \Omega_{1}\right)=\operatorname{Tr}\left(A_{X_{1}} \Omega_{0}\right) \operatorname{Tr}\left(A_{X_{2}} \Omega_{1}\right)-\operatorname{Tr}\left(A_{X_{1}} \Omega_{0} \Omega_{1}^{-1} A_{X_{2}}^{-1}\right) .
$$

The first term on the right hand side is $\left(X_{1}+1\right)\left(X_{2}+1\right)$ and the last term reduces to

$$
-\operatorname{Tr} A_{X_{2}}^{-1} A_{X_{1}} \Omega_{1}=-X_{2}+X_{1}-1 .
$$

Hence

$$
\operatorname{Tr} A_{X_{1}} \Omega_{0} A_{X_{2}} \Omega_{1}=X_{1} X_{2}+2 X_{2}+2=\left(X_{1}+2\right) X_{2}+2=X_{1}^{*} X_{2}^{*}+2 .
$$

The other two cases with $q=2$ are similar (or can be obtained from these by replacing $\gamma$ with $\gamma^{-1}$ ).

Now we do the induction step. Suppose the result true for all products of length less than $q$. We split into three cases.

Case (i) $\Omega_{0}$ appears 3 times consecutively.

After cyclic permutation the product is of the form

$$
A_{X_{1}} \Omega_{0} A_{X_{2}} \Omega_{0} A_{X_{3}} \Omega_{0} \cdots \Omega_{i_{q}} .
$$

We will apply (10), splitting the product as

$$
\left(A_{X_{2}} \Omega_{0}\right) \times\left(A_{X_{3}} \Omega_{0} \cdots \Omega_{i_{q}} A_{X_{1}} \Omega_{0}\right) .
$$

Considering the first term of this split product alone, $A_{X_{2}}$ is still preceded and followed by $\Omega_{0}$. Likewise, taking the second term alone, $A_{X_{1}}$ and $A_{X_{3}}$ are still preceded and followed by the same values of $\Omega_{i}$ as they were before and nothing else has changed. Thus the induction hypothesis gives

$$
\operatorname{Tr} A_{X_{2}} \Omega_{0}=X_{2}^{*} \quad \text { and } \quad \operatorname{Tr} A_{X_{3}} \Omega_{0} \cdots \Omega_{i_{q}} A_{X_{1}} \Omega_{0}=X_{3}^{*} \cdots X_{q}^{*} X_{1}^{*} .
$$

Now consider the remaining term coming from (10):

$$
\operatorname{Tr}\left(A_{X_{2}} \Omega_{0}\left(A_{X_{3}} \Omega_{0} \cdots \Omega_{i_{q}} A_{X_{1}} \Omega_{0}\right)^{-1}\right)=\operatorname{Tr}\left(A_{X_{2}} A_{X_{1}}^{-1} \Omega_{i_{q}}^{-1} \cdots A_{X_{3}}^{-1}\right) .
$$

Cyclically permuting, the three terms $A_{X_{3}}^{-1}, A_{X_{2}}, A_{X_{1}}^{-1}$ combine to give a single term $A_{X_{3}}+X_{2}+X_{1}$, so that the trace has degree at most $q-2$ in the variables $X_{3}+X_{2}+X_{1}$, $X_{4}, \ldots, X_{q}$. Putting all this together proves the claim.

Case (ii) $\Omega_{0}$ appears at most 2 times consecutively. 
Suppose first $q \geq 4$. Thus after cyclic permutation the product is of the form

$$
A_{X_{1}} \Omega_{0} A_{X_{2}} \Omega_{0} A_{X_{3}} \Omega_{1} A_{X_{4}} \cdots \Omega_{1} .
$$

We apply (10) splitting as

$$
\left(A_{X_{1}} \Omega_{0} A_{X_{2}} \Omega_{0} A_{X_{3}} \Omega_{1}\right) \times\left(A_{X_{4}} \cdots A_{X_{q}} \Omega_{1}\right) .
$$

Taking each of these subproducts separately, we see that again the terms $\Omega_{i}$ preceding and following each $A_{X}$ are unchanged. So the induction hypothesis gives

$$
\begin{aligned}
\operatorname{Tr}\left(A_{X_{1}} \Omega_{0} A_{X_{2}} \Omega_{0} A_{X_{3}} \Omega_{1}\right) & =X_{1}^{*} X_{2}^{*} X_{3}^{*}, \\
\operatorname{Tr}\left(A_{X_{4}} \cdots A_{X_{q}} \Omega_{1}\right) & =X_{4}^{*} \ldots X_{q}^{*} .
\end{aligned}
$$

Moreover we note

$$
A_{X_{1}} \Omega_{0} A_{X_{2}} \Omega_{0} A_{X_{3}} \Omega_{1} \Omega_{1}^{-1} A_{X_{q}}^{-1} \cdots A_{X_{4}}^{-1}
$$

is of degree at most $q-2$ in the variables $X_{4}+X_{1}, X_{2}, X_{3}+X_{q}, X_{5}, \ldots, X_{q-1}$. The result follows.

The case $q=3$ is dealt with by splitting

$$
A_{X_{1}} \Omega_{0} A_{X_{2}} \Omega_{0} A_{X_{3}} \Omega_{1} \quad \text { as }\left(A_{X_{3}} \Omega_{1} A_{X_{1}} \Omega_{0}\right) \times A_{X_{2}} \Omega_{0},
$$

using the previously considered case $q=2$, and noting that

$$
A_{X_{3}} \Omega_{1} A_{X_{1}} \Omega_{0} \Omega_{0}^{-1} A_{X_{2}}^{-1}
$$

has degree 1 in the variable $X_{1}+X_{2}+X_{3}$. (Recall that $A_{X}^{-1}=-A_{X}$.)

Case (iii) $\Omega_{0}$ and $\Omega_{1}$ appear alternately.

In this case we split

$$
A_{X_{1}} \Omega_{0} A_{X_{2}} \Omega_{1} A_{X_{3}} \Omega_{0} A_{X_{4}} \cdots \Omega_{1} \quad \text { as } \quad\left(A_{X_{1}} \Omega_{0} A_{X_{2}} \Omega_{1}\right) \times\left(A_{X_{3}} \Omega_{0} A_{X_{4}} \cdots \Omega_{1}\right)
$$

and the argument proceeds in a similar way to that before.

Now we add in the effect of having scc-arcs, that is we deal with the case $h>0$.

Theorem 6.5 Suppose that a matrix product of the form in Proposition 6.4 is modified by the insertion of $h$ blocks $A_{X_{j}} \Omega_{0}^{-1} B_{Y_{r}} \Omega_{0} A_{X_{j}}, r=1, \ldots, h$ for variables $Y_{r} \in \mathbb{C}$. Then its trace is

$$
\pm i^{q}\left(\prod_{r=1}^{h} Y_{r}\right)\left(\prod_{j=1}^{q} X_{j}^{*}\right)+R
$$

where $R$ denotes terms of degree at most $q-2$ in the $X_{j}$. 
Proof We first check the case $q=1, h=1$ by hand. (Note that such a block cannot be the holonomy matrix of a simple closed curve.) We have

$$
A_{X} \Omega_{1} B_{Y} \Omega_{0}=\left(\begin{array}{cc}
X(1+Y)-(X+1) & * \\
* & 1
\end{array}\right),
$$

hence $\operatorname{Tr} A_{X} \Omega_{1} B_{Y} \Omega_{0}=X Y$. Since the term $\Omega_{0} A_{X} \Omega_{1}$ contributes the factor $X$ and the term $B_{Y}$ contributes $Y$, this fulfills our hypothesis.

Now work by induction on $h$. Suppose the result holds for products

$$
\Omega_{u} A_{X_{1}} \Omega_{i_{1}} A_{X_{2}} \Omega_{i_{2}} \cdots A_{X_{s}}
$$

containing at most $h-1$ terms of the form $B_{Y_{r}}$ and consider a product

$$
\Omega_{u} A_{X_{1}} \Omega_{i_{1}} A_{X_{2}} \Omega_{i_{2}} \cdots \Omega_{v} A_{X} \Omega_{1} B_{Y_{h}} \Omega_{0} A_{X} .
$$

There are four possible cases:

$$
u=1, v=0, \quad u=1, v=1, \quad u=0, v=0, \quad u=0, v=1 .
$$

Case $u=1, v=0$ Consider the extra contribution to the trace resulting from the additional block $A_{X} \Omega_{1} B_{Y_{h}} \Omega_{0} A_{X}$. The first occurrence of $A_{X}$ appears in a block $\Omega_{0} A_{X} \Omega_{1}$ which, according to what we want to prove, should contribute a factor $X$. Likewise the block $\Omega_{0} A_{X} \Omega_{1}$ containing the second occurrence of $A_{X}$ should contribute $X$, and the term $B_{Y}$ should contribute $Y$. Thus it is sufficient to show that

$$
\begin{aligned}
& \operatorname{Tr}\left(\Omega_{1} A_{X_{1}} \Omega_{i_{1}} A_{X_{2}} \Omega_{i_{2}} \cdots A_{X_{s}} \Omega_{0} A_{X} \Omega_{1} B_{Y} \Omega_{0} A_{X}\right) \\
& = \pm X^{2} Y \operatorname{Tr}\left(\Omega_{1} A_{X_{1}} \Omega_{i_{1}} A_{X_{2}} \Omega_{i_{2}} \cdots A_{X_{S}} \Omega_{0} A_{X}\right)+R
\end{aligned}
$$

where $R$ denotes terms of total degree at most 2 less then the first term in the $X_{j}$.

Splitting the product as

$$
\left(\Omega_{1} A_{X_{1}} \Omega_{i_{1}} A_{X_{2}} \Omega_{i_{2}} \cdots \Omega_{0} A_{X}\right) \times\left(\Omega_{1} B_{Y} \Omega_{0} A_{X}\right)
$$

and using (10), we see that the second factor contributes $X Y$ and the first factor, containing the sequence $\Omega_{0} A_{X} \Omega_{1}$, contributes $X$. The remaining term

$$
\left(\Omega_{1} A_{X_{1}} \Omega_{i_{1}} A_{X_{2}} \Omega_{i_{2}} \cdots \Omega_{0} A_{X}\right) \times\left(\Omega_{1} B_{Y} \Omega_{0} A_{X}\right)^{-1}
$$

coming from (10) has, as usual, degree in the $X_{j}$ lower by 2 . This proves the claim in this case.

Case $u=1, v=1 \quad$ Again splitting the product as

$$
\left(\Omega_{1} A_{X_{1}} \Omega_{i_{1}} A_{X_{2}} \Omega_{i_{2}} \cdots \Omega_{1} A_{X}\right) \times\left(\Omega_{1} B_{Y} \Omega_{0} A_{X}\right),
$$


the first split factor contains the block $\Omega_{1} A_{X} \Omega_{1}$ which contributes a factor $(X+1)$ to the trace. The second split factor contributes $X Y$.

In the unsplit product we have from the first occurrence of $A_{X}$ the block $\Omega_{1} A_{X} \Omega_{1}$, which contributes a factor $X+1$, and from the second $A_{X}$ the block $\Omega_{0} A_{X} \Omega_{1}$, which contributes $X$, again proving our claim.

Case $u=0, v=0$ This can be done by inverting the previous case. Alternatively, splitting again as

$$
\left(\Omega_{0} A_{X_{1}} \Omega_{i_{1}} A_{X_{2}} \Omega_{i_{2}} \cdots \Omega_{0} A_{X}\right) \times\left(\Omega_{1} B_{Y} \Omega_{0} A_{X}\right),
$$

the first split factor contains the block $\Omega_{0} A_{X} \Omega_{0}$, which contributes a factor $X+1$, while the second split factor contributes, as usual, $X Y$.

In the unsplit product we have from the first $A_{X}$ the block $\Omega_{0} A_{X} \Omega_{1}$ which contributes $X$, and from the second $A_{X}$ the block $\Omega_{0} A_{X} \Omega_{0}$ which contributes $X+1$, again proving our claim.

Case $u=0, v=1 \quad$ Again split as

$$
\left(\Omega_{0} A_{X_{1}} \Omega_{i_{1}} A_{X_{2}} \Omega_{i_{2}} \cdots \Omega_{1} A_{X}\right) \times\left(\Omega_{1} B_{Y} \Omega_{0} A_{X}\right) .
$$

The first split factor, containing $\Omega_{1} A_{X} \Omega_{0}$, contributes $X+2$ and the second split factor contributes $X Y$.

In the unsplit product we have from the first $A_{X}$ the term $\Omega_{1} A_{X} \Omega_{1}$, which contributes $X+1$, and from the second $A_{X}$ the term $\Omega_{0} A_{X} \Omega_{0}$, which contributes $X+1$. The induction still works because, up to terms of lower degree, $X(X+2) Y=$ $(X+1)(X+1) Y$.

Remark 6.6 Not all the cases discussed above are realisable as the holonomy representations of simple connected loops $\gamma$. For example, the cases

$$
v=1, u=1, Y=+2 \text { and } v=0, u=0, Y=-2
$$

produce nonsimple curves.

Proof of Theorem A This follows from Proposition 6.1 on setting $Y_{j}= \pm 2$ in Theorem 6.5. (For the final statement see Lemma 4.3.) 


\section{References}

[1] L Bers, Inequalities for finitely generated Kleinian groups, J. Analyse Math. 18 (1967) 23-41 MR0229817

[2] M Dehn, Lecture notes from Breslau, Archives of the University of Texas at Austin (1922)

[3] A Fathi, F Laudenbach, V Poenaru, editors, Travaux de Thurston sur les surfaces, Astérisque 66-67, Soc. Math. France, Paris (1979) MR568308 Séminaire Orsay, With an English summary

[4] L Keen, C Series, Pleating coordinates for the Maskit embedding of the Teichmüller space of punctured tori, Topology 32 (1993) 719-749 MR1241870

[5] I Kra, Horocyclic coordinates for Riemann surfaces and moduli spaces. I. Teichmüller and Riemann spaces of Kleinian groups, J. Amer. Math. Soc. 3 (1990) 499-578 MR1049503

[6] F Luo, Simple loops on surfaces and their intersection numbers, Math. Res. Lett. 5 (1998) 47-56 MR1618359

[7] S Maloni, The Maskit embedding of a hyperbolic surface, in preparation

[8] A Marden, Outer circles. An introduction to hyperbolic 3-manifolds, Cambridge Univ. Press (2007) MR2355387

[9] B Maskit, Moduli of marked Riemann surfaces, Bull. Amer. Math. Soc. 80 (1974) 773-777 MR0346149

[10] K Matsuzaki, M Taniguchi, Hyperbolic manifolds and Kleinian groups, Oxford Math. Monogr., Oxford Science Publ., The Clarendon Press, Oxford Univ. Press, New York (1998) MR1638795

[11] D Mumford, C Series, D Wright, Indra's pearls. The vision of Felix Klein, Cambridge Univ. Press, New York (2002) MR1913879

[12] R C Penner, J L Harer, Combinatorics of train tracks, Annals of Math. Studies 125, Princeton Univ. Press (1992) MR1144770

[13] C Series, The Maskit embedding of the twice punctured torus arXiv:0808.2119v1

[14] D P Thurston, On geometric intersection of curves on surfaces Available at http:// wWw. math. columbia.edu/ dpt/

Mathematics Institute, University of Warwick

Coventry, CV4 7AL, United Kingdom

s.maloni@warwick.ac.uk, c.m.series@warwick.ac.uk

http://www.warwick.ac.uk/ marhal, http://www.warwick.ac.uk/ masbb/

Received: 15 January 2010 Revised: 25 May 2010 